\title{
Expression analysis of genes related to cold tolerance in Dendroctonus valens
}

\author{
Dongfang Zhao ${ }^{1}$, Chunchun Zheng ${ }^{1}$, Fengming Shi ${ }^{1}$, Yabei Xu ${ }^{1}$, Shixiang Zong ${ }^{1}$, Jing Tao ${ }^{\text {Corresp. } 1}$ \\ ${ }^{1}$ Key Laboratory of Beijing for the Control of Forest Pests, Beijing Forestry University, Beijing, China \\ Corresponding Author: Jing Tao \\ Email address: taojing1029@hotmail.com
}

Pine beetles are well known in North America for their widespread devastation of pine forests. However, Dendroctonus valens LeConte is an important invasive forest pest in China also. Adults and larvae of this bark beetle mainly winter at the trunks and roots of Pinus tabuliformis and Pinus sylvestris; larvae, in particular, result in pine weakness or even death. Since the species was introduced from the United States to Shanxi in 1998, its distribution has spread northward. In 2017, it invaded a large area at the junction of Liaoning, Inner Mongolia and Hebei provinces, showing strong cold tolerance. To identify genes relevant to cold tolerance and the process of overwintering, we sequenced the transcriptomes of wintering and non-wintering adult and larval $D$. valens using the Illumina HiSeq platform. Differential expression analysis methods for other non-model organisms were used to compare transcript abundances in adults and larvae at two time periods, followed by the identification of functions and metabolic pathways related to genes associated with cold tolerance. We detected 4,387 and 6,091 differentially expressed genes (DEGs) between sampling dates in larvae and adults, respectively, and 1,140 common DEGs, including genes encoding protein phosphatase, very long-chain fatty acids protein, cytochrome P450, and putative leucine-rich repeat-containing proteins. In a Gene Ontology (GO) enrichment analysis, 1,140 genes were assigned to 44 terms, with significant enrichment for cellulase activity, hydrolase activity, and carbohydrate metabolism. Kyoto Encyclopedia of Genes and Genomes (KEGG) classification and enrichment analyses showed that the lysosomal and purine metabolism pathways involved the most DEGs, the highly enriched terms included autophagy - animal, pentose and glucuronate interconversions and lysosomal processes. We identified 140 candidate genes associated with cold tolerance, including genes with established roles in this trait (e.g., genes encoding trehalose transporter, fructose-1,6-bisphosphatase, and trehalase). Our comparative transcriptome analysis of adult and larval $D$. valens in different conditions provides basic data for the discovery of key genes and molecular mechanisms underlying cold tolerance. 
1 Expression analysis of genes related to cold tolerance in

2 Dendroctonus valens

3 Dongfang Zhao ${ }^{1}$, Chunchun Zheng ${ }^{1}$, Fengming Shi ${ }^{1}$, Yabei $\mathrm{Xu}^{1}$, Shixiang Zong ${ }^{1}$, Jing Tao ${ }^{1}$

41 Key Laboratory of Beijing for the Control of Forest Pests, Beijing Forestry University, Beijing , China

5

6 Corresponding Author:

7 Jing $\mathrm{TaO}^{1}$

8 No.35 Tsinghua East Road, Haidian District, Beijing, 100083, China

9 Email address: taojing1029@hotmail.com 


\section{Abstract}

Pine beetles are well known in North America for their widespread devastation of pine forests. However, Dendroctonus valens LeConte is an important invasive forest pest in China also. Adults and larvae of this bark beetle mainly winter at the trunks and roots of Pinus tabuliformis and Pinus sylvestris; larvae, in particular, result in pine weakness or even death. Since the species was introduced from the United States to Shanxi in 1998, its distribution has spread northward. In 2017, it invaded a large area at the junction of Liaoning, Inner Mongolia and Hebei provinces, showing strong cold tolerance. To identify genes relevant to cold tolerance and the process of overwintering, we sequenced the transcriptomes of wintering and non-wintering adult and larval $D$. valens using the Illumina HiSeq platform. Differential expression analysis methods for other non-model organisms were used to compare transcript abundances in adults and larvae at two time periods, followed by the identification of functions and metabolic pathways related to genes associated with cold tolerance. We detected 4,387 and 6,091 differentially expressed genes (DEGs) between sampling dates in larvae and adults, respectively, and 1,140 common DEGs, including genes encoding protein phosphatase, very long-chain fatty acids protein, cytochrome $\mathrm{P} 450$, and putative leucine-rich repeat-containing proteins. In a Gene Ontology (GO) enrichment analysis, 1,140 genes were assigned to 44 terms, with significant enrichment for cellulase activity, hydrolase activity, and carbohydrate metabolism. Kyoto Encyclopedia of Genes and Genomes (KEGG) classification and enrichment analyses showed that the lysosomal and purine metabolism pathways involved the most DEGs, the highly enriched terms included autophagy - animal, pentose and glucuronate interconversions and lysosomal processes. We identified 140 candidate genes associated with cold tolerance, including genes with established roles in this trait (e.g., genes encoding trehalose transporter, fructose-1,6-bisphosphatase, and trehalase). Our comparative transcriptome analysis of adult and larval $D$. valens in different conditions provides basic data for the discovery of key genes and molecular mechanisms underlying cold tolerance.

\section{Introduction}

Dendroctonus valens, the red turpentine beetle, is a species of bark beetle that mainly attacks the base of the trunk $P$. tabuliformis. Adults generally lay eggs in the phloem at the base of the trunk or $1.5 \mathrm{~m}$ below the base. After hatching, larvae consume decaying phloem and form a common tunnel. Adults and larvae eat the phloem, destroy the cambium, and cut off nutrient transport in swarms, thereby affecting tree growth or even causing death. This damage reduces the economic and landscape value of the tree (Yan et al., 2005). 
Dendroctonus valens was introduced to Shanxi Province in 1998 and spread rapidly due to the abundant Pinus hosts and warm and dry climate (Sun et al., 2013). The species was introduced to Hebei and Henan in 1999 (Sun et al., 2004), Shaanxi and Qinghai in 2001, and Beijing in 2005, and its distribution continued to expand northward. By 2017, it reached to Chaoyang of Liaoning and Chifeng of Inner Mongolia at approximately $41.5^{\circ} \mathrm{N}$ latitude.

Insect cold tolerance has been studied since the 1960s (Bělehrádek, 1957; Salt, 1961). Research in this area has progressed rapidly since the 1990s, in large part owing to theoretical advances related to insect cold tolerance (Huey et al., 1992; Bale, 2002). Technological and scientific developments have enabled a deeper understanding of cryobiology. Various omics technologies have been used to characterize the molecular mechanisms underlying cold tolerance. Recent studies of cold tolerance in insects have focused on the determination of the supercooling point, survival in low-temperature conditions, the cold tolerance index, and the influence of cold acclimation on insect biology. Transcriptome techniques, such as gene chip technology, expressed sequence tags, serial analysis of gene expression, and RNA sequencing, have been used to identify highly expressed cold-related genes in insects (Barth et al., 2018; Chen et al., 2019; Enriquez \& Colinet, 2019). To screen cold-tolerant genes, a variety of tools for studying differential gene expression are available, including cDNA library screening, suppression subtractive hybridization, and microarray screening (Parker et al., 2018; Martinson et al., 2019; Wang et al., 2019). Two-dimensional electrophoresis, mass spectrometry, nuclear magnetic resonance, and affinity chromatography technology have been used to separate and purify antifreeze proteins extracted from insects, analyze the amino acid composition, and explore the structural characteristics and mechanism of action (Tomalty et al., 2019; Xiang et al., 2020; Graham et al., 2020). Extensive research has confirmed that cold acclimation influences cold tolerance in insects, as determined by a variety of physiological and biochemical methods, such as analyses of the difference between dry and wet weights, differential scanning calorimetry, spectrophotometry, and capillary gas spectroscopy (Holmstrup, 2018; Verheyen et al., 2018; Pei et al., 2020).

In view of the expansion of the distribution of $D$. valens, extensive research has focused on biological characteristics (Liu, Xu \& Sun, 1938; Zhang et al., 2010; Lindeman \& Yack, 2015; Liu et al., 2017), risk analyses (Liu, Xu \& Sun, 1938), biological control ( Storey, Storey \& GuiJun, 2011; Yang, Wang \& Zhang, 2014), symbiotic bacteria (Wang et al., 2012; Raffa, Hanshew \& Mason, 2015; Wang et al., 2017), and pheromones (Chen et al., 2010; Xu, Liu \& Sun, 2014; Kelsey \& Westlind, 2018), but studies of the molecular mechanisms underlying cold tolerance in D. valens are lacking. Other researches on the cold tolerance of bark beetles are mainly reflected in the physiological and biochemical analysis. Many scholars have measured the low temperature survival, supercooling ability, hemolymph osmotic pressure, lipid and carbohydrate 
reserves, and concentration of potential cryoprotectant glycerol of other bark beetles (such as Ips typographus, Pityogenes chalcographus, Dendroctonus armandi, Dendroctonus ponderosae, Pityophthorus juglandis, tiger beetles), the seasonal development of physiological indices underlying gradual acquisition of relatively high cold tolerance to determine the possible overwintering strategies were described (Régnière \& Bentz, 2007; Koštál et al, 2011; Koštál et al, 2014; Wang et al, 2016; Bleiker, Smith \& Humble, 2017; Hefty et al, 2017; Dai et al, 2019; Burns et al, 2020). In addition, previous work has shown that glycerin enhances the cold tolerance of D. ponderosae during overwintering (Bonnett et al, 2012; Fraser et al, 2017). Cold tolerance is an important indicator of the population density, diffusion, and distribution of insects and can explain adaptation to low temperatures in northern regions. Genes related to cold tolerance in insects have been identified in cold-sensitive Drosophila melanogaster (Hoffmann, 2010). A number of candidate genes and proteins related to low-temperature responses (e.g., factors involved in metabolism, the epidermis, cytoskeleton, immune function, and signal transduction (Vesala et al., 2012; Isobe, Takahashi \& Tamura, 2013; Parker et al., 2015) have been reported in drosophila, including heat shock proteins (HSPs) (Colinet et al., 2013; Enriquez \& Colinet, 2019) and Frost genes (Colinet et al., 2013; MacMillan et al., 2016).

Transcriptomics, as a bridge linking genomics and proteomics, has been applied in many fields of biology. It is a powerful tool to evaluate gene expression differences, phylogenetic relationships, rates of protein evolution, genotype-phenotype correlations, chromosome structure, and regulatory mechanisms, as well as for the development of molecular markers and functional genomics research (Oppenheim, 2014). In recent years, with the reduction of sequencing costs, transcriptome sequencing has become increasingly common, particularly RNA-Seq analyses using high-throughput sequencing technology. An obvious advantage of RNA-Seq is the ability to directly assess sequences without prior knowledge of gene structure and to identify novel transcripts. This method enables studies of gene expression in non-model insects and species without sequenced genomes (Malone \& Oliver, 2011; Alvarez, Schrey \& Richards, 2015; Jazayeri, Munoz \& Romero, 2015).

In this study, high-throughput transcriptome sequencing was used to explore key genes and metabolic pathways related to responses to low temperatures in D. valens. These results provide a foundation for further molecular and functional studies of this trait and provide a theoretical reference for the development of a prevention and control strategy.

\section{Materials and Methods}

\section{Experimental insects}

Dendroctonus valens late instar larvae and adults were collected from the field on January 19, 2019, and May 11, 2019, in Heilihe National Nature Reserve, Ningcheng County, Chifeng City, 
Inner Mongolia (Longitude: $118.43^{\circ} \mathrm{E}$; Latitude: $41.41^{\circ} \mathrm{N}$; Altitude: $1050 \mathrm{~m}$ ). Liu Yushan, head of Chifeng Forest Protection Station in Inner Mongolia, has approved sample collection. The average temperature was $-7.36^{\circ} \mathrm{C}$ and $18.82{ }^{\circ} \mathrm{C}$ on these months respectively (http://data.cma.cn/). The samples were stored in liquid nitrogen, brought to the laboratory, and stored at $-80^{\circ} \mathrm{C}$ until RNA extraction.

\section{RNA extraction, cDNA library construction and sequencing}

Total RNA was extracted from $D$. valens collected in January and May using TRIzol reagent (Ambion, Austin, TX, USA) and the RNeasy Plus Mini Kit (No. 74,134; Qiagen, Hilden, Germany) following the manufacturers' instructions. The larvae under cold and normal temperature are referred to as CL and NL respectively, and the adults under cold and normal temperature are referred to as CA and NA respectively. The biological material was whole insect. Each sample consisted of a single individual, and three biological replicates were evaluated per sample. The purity, concentration, and integrity of the total RNA samples were measured using the NanoDrop2000 (IMPLEN, Westlake Village, CA, USA), Agilent 2100 (Agilent Technologies, Santa Clara, CA, USA) and 1.0\% agarose gel electrophoresis. Library construction required a sample of $\geq 2 \mu \mathrm{g}$, a concentration of $\geq 50 \mathrm{ng} / \mu \mathrm{l}$, clear RNA bands, no contamination by impurities (such as pigments, proteins, and sugars), a ratio of $28 / 23 \mathrm{~S}: 18 / 16 \mathrm{~S}$ of $>1, \mathrm{RIN}$ value of $\geq 6.5, \mathrm{OD}_{260 / 280} \geq 1.8$, and $\mathrm{OD}_{260 / 230} \geq 1.5$.

RNA purification, library construction and sequencing were performed at Shanghai Majorbio Bio-pharm Biotechnology Co., Ltd. (Shanghai, China) according to the manufacturer's instructions (Illumina, San Diego, CA). The RNA-seq transcriptome libraries were prepared using Illumina TruSeqTM RNA sample preparation Kit (San Diego, CA). Poly(A) mRNA was purified from total RNA using oligo-dT-attached magnetic beads and then fragmented by fragmentation buffer. Taking these short fragments as templates, double-stranded cDNA was synthesized using random hexamer primers. Then the synthesized cDNA was subjected to endrepair, phosphorylation and 'A' base addition according to Illumina's library construction protocol. Libraries were size selected for cDNA target fragments of 200-300 bp on 2\% low range ultra agarose followed by PCR amplified using Phusion DNA polymerase (New England Biolabs, Boston, MA) for 15 PCR cycles. Subsequently, the library preparations were sequenced on an Illumina Hiseq xten/NovaSeq 6000 sequencer (Illumina, San Diego, CA) for $2 \times 150 \mathrm{bp}$ paired-end reads. All raw sequence data were deposited in the National Center for Biotechnology Information (NCBI) Sequence Read Archive (SRA) database under BioProject accession no. PRJNA609406. The assembled sequences have been submitted to the Gene Expression Omnibus (GEO) with accession number GSE156139. This Transcriptome Shotgun 
Assembly project has been deposited at GenBank under the accession GIWV00000000. The version described in this paper is the first version, GIWV01000000.

\section{Sequence data analysis and assembly}

The Illumina platform converts sequenced image signals into text signals through CASAVA Base Calling and stores these raw data in fastq format. Data for each sample were identified according to the index sequence for subsequent analyses. Fastx_toolkit_0.0.14 (http://hannonlab.cshl.edu/fastx_toolkit/) was used to analyze the base quality, A/T/G/C base content, and base error rate distribution for each sample, as well as the Q20, Q30, and GC contents. SeqPrep (https://github.com/jstjohn/SeqPrep) and Sickle (https://github.com/najoshi/sickle) were used to eliminate raw sequencing data, including adapter sequences, at $3^{\prime}$ end quality reads of $<20$, sequences with a high $\mathrm{N}$ rate of $>10 \%$ (where $\mathrm{N}$ indicates uncertain base information), and sequences of $<30 \mathrm{bp}$ after quality trimming. All sequenced reads after quality control were performed de novo assembly using Trinity_v2.8.5 (https://github.com/trinityrnaseq/trinityrnaseq/wiki). TransRate_v1.0.3 (http://hibberdlab.com/transrate/) was used to filter and optimize the sequences obtained from the de novo assembly of the transcriptome. By the evaluation of common errors, such as chimeras, structural errors, base errors, and incomplete assemblies, a quality score for each contig was obtained, and these were integrated to obtain an overall score for filtering optimization (Smith-Unna et al., 2016). CD-HIT_v4.5.7 (threshold: 0.99) (http://weizhonglilab.org/cd-hit/) was used to remove redundant and similar sequences by a sequence clustering method ( $\mathrm{Li} \&$ Godzik, 2006). The completeness of the assembly was assessed with the Benchmarking Universal Single-Copy Orthologs (BUSCO, version 3.0.2, http://busco.ezlab.org) tool using the metazoa_odb9 (2016-02-13) dataset. BUSCO can identify complete, duplicated, fragmented and missing genes, analyze the completeness of annotated genes and transcriptomes, and perform similar quality comparisons of different data sets (Simão et al ., 2015; Waterhouse et al., 2018).

\section{Functional annotation of genes}

BLAST was used to obtain homologous sequences by searching against several public protein databases, including the NCBI non-redundant $(\mathrm{Nr})$ protein database, the Swiss-Prot protein database, the Protein Family (Pfam) database, the Cluster of Orthologous Groups (COG) database, the GO database (Camacho et al., 2009), and the KEGG database (Kanehisa, 2000). DIAMOND_v0.8.37.99 (E-value: 1e-5) was used for comparisons with the NR library, the annotation of $D$. valens transcript sequences from homologous sequences in other species, and functional annotations of proteins (Buchfink, Xie \& Huson, 2015). BLAST2GO_ 2.5.0 (E-value: 
201 1e-5) was used to evaluate functions of genes and gene products according to the three major 202 GO categories, biological processes, cellular components, and molecular functions (Conesa et al., 2005). KOBAS_2.1.1 (E-value: 1e-5) was used for a KEGG pathway analysis and DIAMOND_v0.8.37.99 (E-value: 1e-5) was used to identify COG groups (Xie et al., 2011).

\section{Differential gene expression analysis}

Bowtie2_2.3.5.1 (https://sourceforge.net/projects/bowtie-bio/files/bowtie2/2.3.5.1/) was used to compare the sequence data after quality control with assembled non-redundant transcriptome sequences. The read counts of a gene in each sample were estimated by mapping clean reads to the Trinity transcripts assembled by RSEM_v1.2.31 (Li \& Dewey, 2011). The Fragments Per Kilobase per Million mapped reads (FPKM) method can be used for standardization to eliminate the effect of gene length and sequencing volume on estimates of expression levels. DESeq2 (Version 1.24.0) and the negative binomial distribution (http://bioconductor.org/packages/stats/bioc/DESeq2/) were used for a statistical analysis to obtain the differentially expressed gene set between wintering periods in D. valens. Differentially expressed genes can be divided into up-regulated and down-regulated genes. The samples collected in May are used as controls, the expression level of a gene in January is stronger than that in May, which is an up-regulated gene, and vice versa. In the differential expression analysis, the Benjamini-Hochberg method was used to correct for multiple testing; the corrected p-value is referred to as p-adjust, and the default value for p-adjust was used as the threshold for screening differentially expressed genes (Benjamini \& Yekutieli, 2001). In particular, the default screening parameters were p-adjust $<0.01$ and $|\log 2 \mathrm{FC}| \geq 1$. FC (fold change) for the identification of a difference in the expression of unigenes between the two groups of samples. The screening results are presented in the form of a volcano plot.

To identify functions associated with the differentially expressed gene set, Goatools_0.6.5 was used to perform a GO enrichment analysis of the genes using Fisher's exact test (Klopfenstein et al., 2018). The Bonferroni method was used to correct p-values. A corrected p-value (FDR) of $<0.05$ indicated significant enrichment for the GO term. To systematically analyze the metabolic pathways involving the differentially expressed genes and the functions of their gene products, Fisher's exact tests were used. Similar to the GO enrichment analysis, when the p-value (FDR) was less than 0.05 , the KEGG pathway was considered significantly enriched in the gene set ( $D u$ et al., 2014; Kanehisa et al., 2017).

\section{Gene expression validation by real-time quantitative PCR}


237

238

239

240

241

242

243

244

245

246

247

248

249

250

251

252

253

254

255

256

257

258

259

260

261

262

263

264

265

266

267

268

269

270

271

genes for larvae and adults, respectively (Zheng et al., 2020). Primers were designed using Primer 3Plus (Untergasser et al., 2007) and synthesized by Ruibo Xingke Biotechnology Co., Ltd. (Beijing, China) (Table S1). A 5× gradient dilution of the cDNA of adults and mature larvae was used as a template to draw the standard curve to determine the amplification efficiency of primers. The target fragment was amplified by conventional PCR and the optimal reaction conditions were explored. The SYBR Green dye method was used for qPCR, and the procedure was performed according to the instructions provided with the Fluorescence Quantitative Reaction Kit (Roche, Basel, Switzerland). The reaction system $(12.5 \mu \mathrm{L})$ included SYBR ${ }^{\circ}$ Premix Ex TaqII $(6.25 \mu \mathrm{L}), 0.5 \mu \mathrm{L}$ of $10 \mu \mathrm{mol} / \mathrm{L}$ forward primer, $0.5 \mu \mathrm{L}$ of $10 \mu \mathrm{mol} / \mathrm{L}$ reverse primer, cDNA $(1 \mu \mathrm{L})$, and $\mathrm{ddH}_{2} \mathrm{O}(4.25 \mu \mathrm{L})$, mixed well on ice. PCR conditions were as follows: $95^{\circ} \mathrm{C}$ for $3 \mathrm{~min}, 40$ cycles at $95^{\circ} \mathrm{C}$ for $10 \mathrm{~s}, 60^{\circ} \mathrm{C}$ for $30 \mathrm{~s}$, and $65^{\circ} \mathrm{C}$ to $95^{\circ} \mathrm{C}$ in increments of $0.5^{\circ} \mathrm{C}$ for $5 \mathrm{~s}$ to generate melting curves. Each reaction was performed for three biological and three technical replicates. The experimental data were normalized by the $2^{-\Delta \Delta t}$ method (Livak \& Schmittgen, 2001). GraphPad Prism 7 was used for statistical analyses and generating plots of real-time PCR results.

\section{Results}

\section{Sequencing and assembly of $D$. valens transcriptomes}

The Illumina sequencing platform was used to sequence 12 samples of $D$. valens. Over $6.1 \mathrm{~Gb}$ of clean data were obtained for each sample, with Q30 $\geq 93.21 \%$, Q20 $\geq 97.82 \%$, and a GC content of $42.98 \%$ (Table 1). Trinity was used to assemble all clean reads from scratch, and 90,404 transcripts were obtained. After clustering and reducing redundancy, 50,677 unigenes were obtained. The average transcript length after assembly was $911.08 \mathrm{bp}$, N50 was 1,803 bp, and the BUSCO score was C:89.2\%[S:84.9\%,D:4.3\%], F:6.9\%, M:3.9\%, n:978 (Table S2). Based on these parameters, the data quality and reliability were high, meeting the requirements for further analyses.

\section{Functional annotation of genes}

The assembled sequences were compared with sequence data in the Nr, Pfam, COG, Swiss-Prot, KEGG, and GO databases using BLAST (e $\leq 10-5)$. Functional annotations were obtained for 28,218 sequences, accounting for approximately $47.86 \%$ of all unigene sequences in the transcriptome (Fig. 1). Among these, 27,047 unigenes were annotated in the $\mathrm{Nr}$ database, accounting for the highest proportion (45.88\%), followed by Pfam (33.84\%), GO (32.22\%), Swiss-Prot (33.32\%), and KEGG (24.94\%). The E-value distribution, identity distribution, and species distribution were used to further analyze homology between Illumina sequences and those in the $\mathrm{Nr}$ database. Some sequences in the samples were contaminated by nematodes, we 
272

273

274

275

276

277

278

279

280

281

282

283

284

285

286

287

288

289

290

291

292

293

294

295

296

297

298

299

300

301

302

303

304

305

306

307

deleted the sequences with nematode contamination and added annotations again. Based on the E-value distribution, $68.99 \%$ of the annotated unigenes $(12,284)$ had very high homology with proteins in the $\mathrm{Nr}$ database (E-value $<1 \mathrm{e}-30$ ), and the other sequences had matches with Evalues ranging from 1e-30 to 1e-5 (Fig. 2A). Further analysis showed that $93.33 \%$ of the sequences had similarities of $>60 \%$ to those in the $\mathrm{Nr}$ database (Fig. 2B). With respect to species, the annotated sequences had the highest degree of match to those of $D$. ponderosae, with 12,772 sequences and a matching rate of $71.73 \%$ (Fig. 2C).

\section{Differentially expressed gene screening results of $D$. valens under different temperature conditions}

The results showed that 4,387 DEGs were found among the larvae sampled in January and May. Compared with larvae collected in May, 24,85 (56.64\%) of genes collected in January were upregulated, 1,902 (43.36\%) were down-regulated (Fig. 3A). There were 6,091 DEGs in adults, including 3,670 (60.25\%) up-regulated genes and 2,421 (39.75\%) down-regulated genes in January compared with May (Fig. 3B).

As shown in a Venn diagram in Fig. 4, 1,140 genes were differentially expressed between January and May in both larvae and adults (Table S3), 4,951 genes were differentially expressed only in adults, and 3,247 genes were differentially expressed only in larvae. Among the common DEGs in adults and larvae, 218 lacked functional annotations in any database, 505 were downregulated in adults and larvae, and 370 were up-regulated. In addition, 168 DEGs were upregulated in larvae and down-regulated in adults, only 97 DEGs were down-regulated in larvae and up-regulated in adults. We speculated that the common DEGs in adults and larvae under different field temperatures might play an important role in the response to low temperatures; accordingly, we focused on these genes in further functional analyses.

\section{Classification of common DEGs}

The 1,140 common DEGs were assigned to three GO domains: biological process (BP), cellular component (CC), and molecular function (MF), involving 44 total GO terms (Fig. 5). In the BP category, the most frequent terms were cellular process (348 genes), followed by metabolic process ( 275 genes). In the $\mathrm{CC}$ category, the most frequent terms were cell part and membrane part (371 and 250, respectively). In the MF category, with binding accounting for the largest proportion of genes (408 genes). A KEGG analysis revealed that 1,421 unigenes belonged to 287 pathways (Table 2). There were 19 metabolic pathways containing 10-20 genes, and the remaining pathways all had fewer than 10 genes. The pathways identified in this analysis were mainly involved in the processes of translation, endocrine system, transport catabolism, carbohydrate metabolism, signal transduction, nucleotide metabolism, and cell growth and death, 
308

309

310

311

312

313

314

315

316

317

318

319

320

321

322

323

324

325

326

327

328

329

330

331

332

333

334

335

336

337

338

339

340

341

342

343

indicating that signal transduction and substance transport activities may be important in $D$. valens.

\section{Enrichment analysis of common DEGs}

In an evaluation of the top 25 common DEGs (Table 3), 12 terms in BP and 13 terms in MF were significantly enriched. The entry carbohydrate metabolic process (GO: 0005975) was significantly enriched in $\mathrm{BP}$, indicating that carbohydrate metabolism in $D$. valens might contribute to overwintering. In the MF category, the two most significantly enriched GO terms were hydrolase activity, acting on glycosyl bonds (GO: 0016798) and hydrolase activity, hydrolyzing O-glycosyl compounds (GO: 0004553), further illustrating that the molecular activity of $D$. valens is strong under low temperatures. Among the common up-regulated DEGs between sampling dates in adults and larvae, the top two were nucleic acid binding (GO:0003676), RNA metabolic process (GO:0016070) (Fig. S1-A). Among the common downregulated DEGs, carbohydrate metabolic process (GO: 0005975), hydrolase activity, acting on glycosyl bonds (GO: 0016798), hydrolase activity, hydrolyzing O-glycosyl compounds (GO: 0004553), cellulase activity (GO: 0008810), catalytic activity(GO:0003824) and other terms were significantly enriched (Fig. S1-B).

A KEGG analysis revealed that common DEGs could be classified into 287 pathways, the highly enriched terms included pentose and glucuronate interconversions (map00040), lysosome (map04142), and autophagy-animal (map04140), which belonged to carbohydrate metabolism and transport and catabolic metabolism pathways, respectively. It is possible that these processes contribute to overwintering in D. valens. Among the common up-regulated DEGs between sampling dates in adults and larvae, we detected significant enrichment for homologous recombination (map03440) and basal transcription factors (map03022) (Fig. S2-A). The enrichment results for common down-regulated DEGs in adults and larvae were similar to common DEGs for adults and larvae, including lysosome (map04142), pentose and glucuronate interconversions (map00040), galactose metabolism (map00052), and other terms (Fig. S2-B).

\section{Screening of genes related to cold tolerance among common DEGs}

Based on previous studies of insect cold tolerance and the keywords obtained in manual searches of the annotation information for the common DEG set, we selected 140 candidate genes related to cold tolerance. In total, 72 genes encoded enzymes and proteins related to the synthesis and metabolism of cryoprotectants, including low-molecular-weight polyols, trehalose, and lowmolecular-weight amino acids (Fig. 6).

In addition, two genes encoding the cytoprotective protein $H S P, 12$ genes encoding cytoskeletal proteins, 9 genes involved in repair, detoxicant, antioxidant defense, 10 genes 
344

related to signal transduction, 1 gene related to cell process regulation, and 32 genes associated with other processes related to cold tolerance were annotated (Table 4).

\section{Expression level verification}

We selected 14 candidate genes with potential roles in cold tolerance, including 11 annotated genes described in Table 4 and Fig. 6, and three genes with high differential expression in the common DEG set but without functional annotation. Fourteen genes encoding staphylococcal nuclease domain-containing protein 1 (TRINITY_DN22243_c0_g1), ATP synthase subunit alpha (TRINITY_DN18757_c0_g2), protein phosphatase 1A (TRINITY_DN26187_c0_g1), fructose-1,6-bisphosphatase 1 (TRINITY_DN21780_c1_g1), glyceraldehyde-3-phosphate dehydrogenase 2 (TRINITY_DN22610_c0_g2), putative glutamate synthase (TRINITY_DN27207_c0_g1), cathepsin L1 (TRINITY_DN18927_c0_g1), probable lowspecificity L-threonine aldolase 2 (TRINITY_DN22377_c1_g1), serine/threonine-protein kinase (TRINITY_DN26090_c0_g1), fatty acid desaturase (TRINITY_DN19691_c0_g1), E3 ubiquitinprotein ligase (TRINITY_DN25425_c0_g4) and three genes annotated to unknown or uncharacterized proteins (TRINITY_DN27537_c0_g1, TRINITY_DN20229_c0_g1, and TRINITY_DN22439_c0_g5) were evaluated by qPCR (Fig. 7 and Fig. 8).

Based on the qPCR results, the expression differences in the 14 DEGs were consistent with those detected by RNA-seq, indicating that the sequencing data were accurate and reliable and can be used as a reference for subsequent research. Slight differences in fold change values may be due to operational errors and the different principles of the analysis methods for transcriptome sequencing and qPCR.

\section{Discussion}

\section{Transcriptome sequencing quality and analysis of differentially expressed genes}

Based on searches against the Nr protein database of NCBI, 27,047 genes (45.88\%) were homologous to known sequences, indicating that the transcriptome includes a large number of new genes with unknown functions. These genes may be related to the unique biological characteristics and environmental adaptability of the species. Taking May data as a control, comparing January data with May data, the result showed the 1,140 common DEGs between sampling dates in both larvae and adults, 370 were up-regulated in both adults and larvae and 505 were down-regulated, similar to the results of transcriptome analyses of the Chinese white wax scale insect (Ericerus pela) and the desert tenebrionid beetle (Microdera punctipennis), although the frequency of upregulated genes is low, these results suggest that some physiological activities remain active or are initiated in but no longer in May (Yu et al., 2016; Tusong et al., 2017). 
The 370 up-regulated genes in both adult and larvae encoded several proteins that may be related to cold tolerance, including protein phosphatase, elongation of very long chain fatty acids protein, E3 SUMO-protein ligase, cytochrome $\mathrm{P} 450$, and putative leucine-rich repeat-containing protein. Studies show that under low-temperature and freeze-thaw conditions, protein phosphorylation and dephosphorylation are important regulatory mechanisms related to many metabolic functions. Protein kinases and phosphatases can regulate the activity of many transcription factors and participate in the differential expression of genes involved in cold and freeze resistance (Pfister \& Storey, 2006). Overwintering insects need to neutralize or prevent the production of harmful metabolites. To modify cytotoxic metabolites, cytochrome $\mathrm{P} 450$ in the Antarctic midge (Belgica antarctica) is up-regulated during recovery from dehydration (Benoit et al., 2009). We speculate that cytochrome P450 plays a similar role in cold tolerance. In addition, elongation of very long chain fatty acids protein and leucine-rich repeat-containing protein contribute to the synthesis and metabolism of low-molecular-weight cryoprotectants, and other genes may be important for the maintenance of dormancy and related physiological processes.

We identified 1,140 unigenes with differential expression in both adults and larvae, accounting for only $18.72 \%$ of the DGEs in adults and $25.99 \%$ of the DGEs in larvae. Most of the DGEs were not expressed in adults and larvae but were involved in the synthesis and expression of adaptation-related genes at low temperatures, such as some heat shock proteins, the sugar transporter SWEET1, and calcium channel proteins. For cell survival, heat shock proteins are important for survival during the winter. Many insects alleviate stress by upregulating HSP (Storey \& Storey, 2011; King \& Macrae, 2015; Toxopeus, Koštál \& Sinclairet, 2019; Li, Wang \& Jiang, 2019), but only a subset of HSP genes are up-regulated, consistent with previous results (Zhang et al., 2011). In this study, HSP genes were up-regulated in both adult and larvae, including $h s p 20, h s p 70$, and $h s p 90$. The differentially co-expressed gene set only one $h s p 70$ and one $h s p 68$. Although the specific function of $h s p 70$ in survival at low temperatures in insects is not clear, it improves the response to stress by re-folding damaged proteins and redissolving insoluble proteins (Craig et al.,1985) as well as marking irreparable proteins for degradation (Terlecky et al., 1992). The sugar transporter SWEET1 is a cold tolerance gene based on a transcriptome analysis of the Asian lady beetle (Harmonia axyridis) at normal and low temperatures (Tang et al., 2017). In larvae, calcium channel proteins are significantly upregulated compared to levels in adults, and calcium signaling pathways are also important in cold stress signaling (Reddy et al., 2011; Denlinger et al., 2013; Wang et al., 2013).

Most of Duman's work (as well as Koštál's) has centered on freeze avoiding species that deeply supercool - hence, the interest in antifreeze proteins by these authors. For lots of other species, however, freeze tolerance is the adaptation used (Koštál et al., 2011; Duman, 2015). 
415 However, anti-freeze proteins (AFPs) were not included in our significant DEG sets. It may be

416 due to $D$. valens is a freezing-tolerant species by using the freeze tolerance strategy for subzero

417 survival. It can permit extracellular ice formation within tissues and actively form ice-nucleating

418 proteins (Zhao, Yang \& Gregoire, 2009). It is possible that the relationship between transcript

419 and protein levels is complex, and transcriptome and proteome analyses often show weak

420 correlations between transcript and protein level (Cui, 2017).

421

\section{Analysis of genes related to cold tolerance among common DEGs}

A large number of DEGs were related to the synthesis and metabolism of small cryoprotectants. Freezing-tolerant insects accumulate low-molecular-weight cryoprotectants, such as polyols (e.g., glycerol), sugars (e.g., trehalose), or amino acids (e.g., proline), to confer freezing tolerance. Elevated proline concentrations in the body can improve the survival of cold-sensitive D. melanogaster larvae at low temperatures, and trehalase plays a role in regulating the cold tolerance in H. axyridis and New Zealand alpine insects (Imek et al., 2012; Wharton, 2011;

Toxopeus et al., 2017). In our results, there are seven facilitated trehalose transporter Tretl genes differentially expressed (Fig. 6). The correlation between cold tolerance and upregulation of trehalose transporters has been demonstrated in the cold-acclimated spring field cricket (Gryllus veletis) transcriptome. It is speculated that the Tret-1 promotes trehalose export from the fat body into the hemolymph during acclimation, resulting in hemolymph trehalose accumulation (Toxopeus \& Sinclairet, 2019). Pyrroline-5-carboxylate reductase is very important in the synthesis of proline. Studies have shown that D. melanogaster, the Antarctic midge ( $B$. antarctica) and New Zealand stick insects up-regulate the pyrroline-5-carboxylate reductase in association with proline accumulation (MacMillan et al., 2016; Teets et al., 2012; Dennis et al., 2015). Fatty acid synthase-related genes are differentially expressed at low temperatures, it has been showned that the production of fatty acids maintained the homoeoviscosity of the cellular membranes, which contributes to enhanced cold tolerance (Kayukawa et al., 2007; Michaud \& Denlinger, 2007; Goto et al., 2010).

A number of genes we observed in $D$. valens have been suggested as contributing to the cold tolerance of insects including cathepsin (Dennis et al., 2015), fructose-2,6-bisphosphatase (Fraser et al., 2017), pancreatic triacylglycerol lipase (Bonnett et al., 2012; Fraser et al., 2017), zinc finger proteins (Xu et al., 2017), glyceraldehyde-3-phosphate dehydrogenase 2 (Fraser et al., 2017), NADH dehydrogenase (Dunning et al., 2013), ATP synthase subunit alpha ( $L i \&$ Denlinger, 2018), elongation factor (Tusong et al., 2017), staphylococcal nuclease domaincontaining protein 1 (Dunning et al., 2013), etc. We hypothesize that changes in the expression of these genes increase the cold tolerance of $D$. valens. 
450

451

452

In this study, 12 cytoskeletal genes were differentially expressed (e.g., Tubulin, Actin, and Myosin; Table 4), and cytoskeletal genes have been identified to play an important role in adaptation to low temperature in many other insects such as the northern house mosquito (Culex pipiens) (Kim et al., 2006), the drosophilid fly (Chymomyza costata) (Stetina et al., 2018), M. punctipennis (Tusong et al., 2017), D. melanogaster (MacMillan et al., 2016), the fall field cricket (Gryllus pennsylvanicus) (Des Marteaux et al., 2017). We speculate that the differential expression of cytoskeletal genes are necessary particularly for maintaining cell integrity during the wintering period. In addition, studies have shown that the drosophilid fly (C. costata) (Poupardin et al., 2015), the spring field cricket (G. veletis) (Toxopeus \& Sinclairet, 2019) and the flesh fly (Sarcophaga bullata) (Teets et al, 2012) upregulate some signaling genes after cold treatment. The expression of some signal transduction-related genes in D. valens (such as guanine nucleotide-binding protein, $\mathrm{Ca}^{2+}$ channel, $\mathrm{G}$ kinase-anchoring protein, G-protein coupled receptor Mth2; Table 4 ) were altered with temperature, but it is difficult to predict how these changes enhance cold cold tolerance.

In addition, low-molecular-weight cryoprotectants are related to anabolism genes, cytoskeleton protein genes, and repair-related genes (Toxopeus \& Sinclair, 2018), in addition to many unannotated genes. Although the specific mechanism of action of these genes in insects is unclear, they may also play an important role in cold resistance. Cold tolerance in insects is a complex process, involving interactions among multiple genes (Toxopeus \& Sinclair, 2018).

DEGs involved in cold tolerance are expected to be up-regulated under cold stress; however, most DEGs in our study were down-regulated. One is that the metabolic rate of D. valens in January may be much lower than in May. Studies have shown that when spring becomes warmer, almost all enzymes involved in carbohydrate metabolism (glycolysis, citric acid cycle, glycerol biosynthesis), oxidative stress tolerance, detoxification mechanism, cryoprotectant metabolism protein levels decrease than winter, the strong winter metabolic suppression inhibition can improve the supercooling ability, which has been verified in the overwintering mountain pine beetles (Lester \& Irwin, 2012; Bonnett et al., 2012; Robert et al., 2016). Second, it is possible that genes and proteins related to low temperature adaptation were upregulated at the beginning of the winter and that expression dropped when they were no longer needed, with the saturation of expression levels in late January (Fraser et al., 2017). Therefore, protein expression levels should be a focus of future research.

\section{Cold tolerance-associated GO and KEGG enrichment analysis}

GO terms that were significantly enriched in the down-regulated gene set were carbohydrate metabolism processes, hydrolase activity, cellulase activity, catalytic activity and so on. It is similar to the GO enrichment results of the differentially expressed genes of the European spurge 
486

487

488

489

490

491

492

493

494

495

496

497

498

499

500

501

502

503

504

505

506

507

508

509

510

511

512

513

514

515

516

517

518

519

520

hawkmoth (Hyles euphorbiae) under cold treatment (Barth et al., 2018). Under stress, metabolic processes are down-regulated in many organisms, and the down-regulation of hydrolase and cellulase activity may be the result of metabolic down-regulation. The downregulation of carbohydrate metabolism suggests that the relationship between energy utilization and temperature is altered in insects during the winter by changes in thermal sensitivity and the inhibition of metabolic rates; these insects adapt to low temperature by changing energy metabolism (Storey \& Storey 1990; Sinclair, 2015).

In the co-expressed gene set, a KEGG enrichment analysis showed enrichment for pentose and glucuronate interconversions, lysosome, autophagy - animal, and so on. The autophagy pathway shows that $D$. valens activates its own protective mechanism to repair and clear damaged cells under stress to maintain body operation. We hypothesize that the ability of $D$. valens to clear cold damaged cells may increase in winter (Allen \& Baehrecke 2020).

The pathways basal transcription factors and homologous recombination were significantly enriched in the up-regulated gene set. Studies have shown that RNA polymerase II-dependent transcription involves the regulation of basic transcription factors, and RNA polymerases I, II, and III are also up-regulated in the transcripts of cold-acclimated G. veletis (Toxopeus \& Sinclairet, 2019). Some significantly enriched pathways (pentose and glucuronate interconversions, galactose metabolism, fatty acid elongation, citrate cycle (TCA cycle), lysosome, and autophagy - animal) were detected for common down-regulated DEGs. Similar results were obtained in the transcriptome response to cold stress of the ladybird (Cryptolaemus montrouzieri) (Zhang et al., 2015) and Galeruca daurica (Joannis) (Zhou et al., 2019). These metabolic pathways indicated that overwintering D. valens may produce or consume less energy, consistent with the general response of winter insects to low temperatures by inhibiting the metabolic rate (Sinclair, 2015).

In conclusion, we used RNA-Seq technology to analyze the $D$. valens transcriptome at different periods in the field. We identified many common genes and pathways with potentially important roles in overwintering, improving our understanding of the molecular basis for survival in low temperatures. This study provides an overview of candidate genes associated with cold tolerance in insects, and further validation and functional analyses are needed. Our data will facilitate further molecular studies of cold tolerance in D. valens and provide new insights into insect adaptation to harsh environments.

\section{Conclusions}

In a comparative transcriptome analysis of D. valens in January and May, we used the samples collected in May as controls and detected 4,387 and 6,091 DEGs in larvae and adults, 
521 respectively, including 1,140 genes that differentially expressed at both stages, among which

522538 genes were up-regulated and 602 genes were down-regulated in larvae; in adults, 467 genes

523 were up-regulated and 673 genes were down-regulated. During the overwintering process, one

524 strategy for survival in low temperatures is the synthesis of low-molecular-weight antifreeze

525 substances, anti-oxidative stress factors, molecular chaperones, and signal transduction factors.

526 The DEGs were enriched for the GO terms cellulase activity, hydrolase activity, and

527

carbohydrate metabolism and for the lysosomal and pentose and glucuronate interconversions

528

metabolic pathways. We identified 140 genes that may be related to cold tolerance in the

529

common DEGs, some of which are associated with cold tolerance based on previous studies. The

530

results provided basic data for the subsequent discovery of key genes for cold tolerance in $D$.

531 valens and the discovery of molecular mechanisms underlying this trait.

532

533

534

535

536

537

538

539

540

541

542

543

544

545

546

547

548

549

550

551

552

553

\section{Acknowledgments}

The data were analyzed on the free online platform of Majorbio Cloud Platform

(www.majorbio.com), Qingqing Liu and Ling Wang provided professional service. Field work of sample collection was partially supported by the Heilihe National Nature Reserve, Chifeng, Inner Mongolia, China.

\section{ADDITIONAL INFORMATION AND DECLARATIONS}

\section{Funding}

This research was funded by "Chinese National Natural Science Foundation" (NO. 31870642).

The funders had no role in study design, data collection and analysis, decision to publish, or preparation of the manuscript.

\section{Grant Disclosures}

The following grant information was disclosed by the authors:

Chinese National Natural Science Foundation: 31870642.

\section{Competing Interests}

The authors declare no conflict of interest.

\section{Author Contributions}

- Dongfang Zhao conceived and designed the experiments, performed the experiments, analyzed the data, contributed reagents/materials/analysis tools, wrote original draft preparation, prepared figures and/or tables, reviewed drafts of the paper.

- Chunchun Zheng performed the experiments, contributed reagents/materials/analysis tools.

- Fengming Shi performed the experiments, contributed reagents/materials/analysis tools. 
- Yabei Xu conceived and designed the experiments.

- Jing Tao and Shixiang Zong conceived and designed the experiments, reviewed drafts of the paper, approved the final draft.

\section{DNA Availability}

The following information was supplied regarding data availability:

The National Center for Biotechnology Information (NCBI) Sequence Read Archive (SRA) database (BioProject accession number: PRJNA609406).

The National Center for Biotechnology Information (NCBI) Gene Expression Omnibus (GEO) database (accession number GSE156139).

This Transcriptome Shotgun Assembly project has been deposited at GenBank under the accession GIWV00000000.

\section{Supplemental Information}

Supplemental information for this article can be found online at http://dx.doi.org/10.7717/.

\section{References}

Allen EA, Baehrecke EH. 2020. Autophagy in animal development. Cell Death \& Differentiation DOI 10.1038/s41418-020-0497-0.

Alvarez M, Schrey AW, Richards CL. 2015. Ten years of transcriptomics in wild populations: what have we learned about their ecology and evolution? Molecular Ecology 24:710-725 DOI 10.1111/mec.13055.

Bale JS. 2002. Insects and low temperatures: from molecular biology to distributions and abundance. Philosophical Transactions: Biological Sciences 357:849-862 DOI 10.1098/rstb.2002.1074.

Barth MB, Buchwalder K, Kawahara AY, Zhou X, Liu S, Krezdorn N, Rotter B, Horres R, Hundsdoerfer AK. 2018. Functional characterization of the Hyles euphorbiae hawkmoth transcriptome reveals strong expression of phorbol ester detoxification and seasonal cold hardiness genes. Frontiers in Zoology 15:20 DOI 10.1186/s12983-018-0252-2.

Belehradek J. 1957. Physiological aspects of heat and cold. Annual Review of Physiology 19:5982 DOI 10.1146/annurev.ph.19.030157.000423.

Benjamini Y, Yekutieli D. 2001. The control of the false discovery rate in multiple testing under dependency. The Annals of Statistics 29:1165-1188 DOI 10.1214/aos/1013699998.

Benoit JB, Jr Lee RE, Lopez-Martinez G, Rinehart JP, Denlinger DL, Elnitsky MA. 2009. Dehydration, rehydration, and overhydration alter patterns of gene expression in the Antarctic midge, Belgica antarctica. Journal of Comparative Physiology, B. Biochemical, Systemic, and Environmental Physiology 179:481-491 DOI 10.1007/s00360-008-0334-0. 
Bleiker KP, Smith GD, Humble LM. 2017. Cold Tolerance of Mountain Pine Beetle (Coleoptera: Curculionidae) Eggs From the Historic and Expanded Ranges. Environmental Entomology 46:1165-1170 DOI 10.1093/ee/nvx127.

Bonnett TR, Robert JA, Pitt C, Fraser JD, Keeling CI, Bohlmann J, Huber DPW. 2012. Global and comparative proteomic profiling of overwintering and developing mountain pine beetle, Dendroctonus ponderosae (Coleoptera: Curculionidae), larvae. Insect Biochemistry and Molecular Biology 42:890-901 DOI 10.1016/j.ibmb.2012.08.003.

Buchfink B, Xie C, Huson D. 2015. Fast and sensitive protein alignment using DIAMOND. Nature Methods 12:59-60 DOI 10.1038/nmeth.3176.

Burns M, Herrera D, Brosius T, Muir TJ. 2020. Depressed supercooling point and increased glycerol concentration in overwintering adult tiger beetles (Cicindelida). Cryoletters 41:216-222.

Camacho C, Coulouris G, Avagyan V, Ma N, Papadopoulos J, Bealer K, Madden TL. 2009. BLAST+: architecture and applications. Bmc Bioinformatics 10:421 DOI 10.1186/14712105-10-42.

\section{Chen K, Tang T, Song Q, Wang Z, He K, Liu X, Song J, Wang L, Yang Y, Feng C. 2019.} Transcription analysis of the stress and immune response genes to temperature stress in Ostrinia furnacalis. Frontiers in Physiology 10:1289 DOI 10.3389/fphys.2019.01289.

Colinet H, Siaussat D, Bozzolan F, Bowler K. 2013. Rapid decline of cold tolerance at young age is associated with expression of stress genes in Drosophila melanogaster. The Journal of Experimental Biology 216:253-259 DOI 10.1242/jeb.076216.

Conesa A, Gotz S, Garcia-Gomez JM, Terol J, Talon M, Robles M. 2005. Blast2GO: a universal tool for annotation, visualization and analysis in functional genomics research. Bioinformatics 21:3674-3676 DOI 10.1093/bioinformatics/bti610.

Craig EA, Craig EA, Schlesinger MJ, Schlesinger MJ. 1985. The Heat Shock Respons. Critical Reviews in Biochemistry 18:239-280 DOI 10.3109/10409238509085135.

Cui M, Hu P, Wang T, Tao J, Zong S. 2017. Differential transcriptome analysis reveals genes related to cold tolerance in seabuckthorn carpenter moth, Eogystia hippophaecolus. PLoS One 12:e187105 DOI 10.1371/journal.pone.0187105.

Dai L, Zheng J, Wang Y, Sun Y, Chen H. 2019. Survival physiology and sex ratio of the Chinese white pine beetle Dendroctonus armandi (Coleoptera: Scolytinae) during host colonization and overwintering. Bulletin of Entomological Research 110:115-122 DOI $10.1017 / \mathrm{s} 0007485319000361$.

Denlinger DL, Yi SX, Teets NM, Lee Jr RE. 2013. Calcium signaling mediates cold sensing in insect tissues. Proceedings of the National Academy of Sciences of the United States of America. 110:9154-9159 DOI 10.1073/pnas.1306705110. 
624

625

626

627

628

629

630

631

632

633

634

635

636

637

638

639

640

641

642

643

644

645

646

647

648

649

650

651

652

653

654

655

656

657

658

659

Dennis AB, Dunning LT, Sinclair BJ, Buckley TR. 2015. Parallel molecular routes to cold adaptation in eight genera of New Zealand stick insects. Scientific Reports 5:13965 DOI 10.1038/srep13965.

Des Marteaux LE, McKinnon AH, Udaka H, Toxopeus J, Sinclair BJ. 2017. Effects of coldacclimation on gene expression in Fall field cricket (Gryllus pennsylvanicus) ionoregulatory tissues. BMC Genomics 18(1) DOI 10.1186/s12864-017-3711-9.

Du J, Yuan Z, Ma Z, Song J, Xie X, Chen Y. 2014. KEGG-PATH: Kyoto encyclopedia of genes and genomes-based pathway analysis using a path analysis model. Molecular BioSystems 10:2441-2447 DOI 10.1039/c4mb00287c.

Duman JG. 2015. Animal ice-binding (antifreeze) proteins and glycolipids: an overview with emphasis on physiological function. The Journal of Experimental Biology 218:1846-1855 DOI 10.1242/jeb.116905.

Dunning LT, Dennis AB, Park D, Sinclair BJ, Newcomb RD, Buckley TR. 2013. Identification of cold-responsive genes in a New Zealand alpine stick insect using RNA-Seq. Comparative Biochemistry and Physiology Part D: Genomics and Proteomics 8:24-31 DOI 10.1016/j.cbd.2012.10.005.

Enriquez T, Colinet H. 2019. Cold acclimation triggers major transcriptional changes in Drosophila suzukii. Bmc Genomics 20:413 DOI 10.1186/s12864-019-5745-7.

Fraser JD, Bonnett TR, Keeling CI, Huber DPW. 2017. Seasonal shifts in accumulation of glycerol biosynthetic gene transcripts in mountain pine beetle, Dendroctonus ponderosae Hopkins (Coleoptera: Curculionidae), larvae. PeerJ 5:e3284 DOI 10.7717/peerj.3284.

Goto SG, Udaka H, Ueda C, Katagiri C. 2010. Fatty acids of membrane phospholipids in Drosophila melanogaster lines showing rapid and slow recovery from chill coma. Biochemical and Biophysical Research Communications 391:1251-1254 DOI 10.1016/j.bbrc.2009.12.053.

Graham LA, Boddington ME, Holmstrup M, Davies PL. 2020. Antifreeze protein complements cryoprotective dehydration in the freeze-avoiding springtail Megaphorura arctica. Scientific Reports 10:3047 DOI 10.1038/s41598-020-60060-Z.

Hefty AR, Seybold SJ, Aukema BH, Venette, RC. 2017. Cold Tolerance of Pityophthorus juglandis (Coleoptera: Scolytidae) From Northern California. Environmental Entomology 46:967-977 DOI 10.1093/ee/nvx090.

Hoffmann AA. 2010. Physiological climatic limits in Drosophila: patterns and implications. The Journal of Experimental Biology 213:870-880 DOI 10.1242/jeb.037630.

Holmstrup M. 2018. Screening of cold tolerance in fifteen springtail species. Journal of Thermal Biology 77:1-6 DOI 10.1016 / j.jtherbio.2018.07.017.

Huey RB, Crill WD, Kingsolver JG, Weber KE. 1992. A method for rapid measurement of 
660

661

662

663

664

665

666

667

668

669

670

671

672

673

674

675

676

677

678

679

680

681

682

683

684

685

686

687

688

689

690

691

692

693

694

695

heat or cold resistance of small insects. Functional Ecology 6:489-494 DOI $10.2307 / 2389288$.

Imek P, Zahradní Ková H, Cimlová J, Tětina T, Ko Tál V. 2012. Conversion of the chill susceptible fruit fly larva (Drosophila melanogaster) to a freeze tolerant organism. Proceedings of the National Academy of Sciences of the United States of America 109:3270-3274 DOI 10.1073/pnas.1119986109.

Isobe K, Takahashi A, Tamura K. 2013. Cold tolerance and metabolic rate increased by cold acclimation in Drosophila albomicans from natural populations. Genes \& Genetic Systems 88:289-300 DOI 10.1266/ggs.88.289.

Jazayeri SM, Munoz LMM, Romero HM. RNA-Seq: a glance at technologies and methodologies. Acta Biológica Colombiana 20:23-35 DOI 10.15446/abc.v20n2.43639.

Kanehisa M, Furumichi M, Tanabe M, Sato Y, Morishima K. 2017. KEGG: new perspectives on genomes, pathways, diseases and drugs. Nucleic Acids Research 45:D353D361 DOI 10.1093/nar/gkw1092.

Kanehisa M. 2000. KEGG: kyoto encyclopedia of genes and genomes. Nucleic Acids Research 28:27-30 DOI 10.1093/nar/28.1.27.

Kayukawa T, Chen B, Hoshizaki S, Ishikawa Y. 2007. Upregulation of a desaturase is associated with the enhancement of cold hardiness in the onion maggot, Delia antiqua. Insect Biochemistry and Molecular Biology 37:1160-1167 DOI 10.1016/j.ibmb.2007.07.007.

Kelsey RG, Westlind DJ.2018. Attraction of red turpentine beetle and other S colytinae to ethanol, 3-carene or ethanol+3-carene in an O regon pine forest. Agricultural and Forest Entomology 20(2): 272-278 DOI 10.1111/afe.12257.

Kim M, Robich RM, Rinehart JP, Denlinger DL. 2006. Upregulation of two actin genes and redistribution of actin during diapause and cold stress in the northern house mosquito, Culex pipiens. Journal of Insect Physiology 52:1226-1233 DOI 10.1016/j.jinsphys.2006.09.007.

King AM, MacRae TH. 2015. Insect heat shock proteins during stress and diapause. Annual Review of Entomology 60:59-75 DOI 10.1146/annurev-ento-011613-162107.

Klopfenstein DV, Zhang L, Pedersen BS, Ramirez F, Vesztrocy AW, Naldi A, Mungall CJ, Yunes JM, Botvinnik O, Weigel M, Dampier W, Dessimoz C, Flick P, Tang H. 2018. GOATOOLS: A Python library for Gene Ontology analyses. Scientific Reports 8:10872 DOI 10.1038/s41598-018-28948-z.

Koštál V, Doležal P, Rozsypal J, Moravcová M, Zahradníčková H, Šimek P. 2011. Physiological and biochemical analysis of overwintering and cold tolerance in two Central European populations of the spruce bark beetle, Ips typographus. Journal of Insect Physiology 57:1136-1146 DOI 10.1016/j.jinsphys.2011.03.011. 
696

Koštál V, Korbelová J, Rozsypal J, Zahradníčková H, Cimlová J, Tomčala A, Šimek P. 2011. Long-term cold acclimation extends survival time at $0^{\circ} \mathrm{C}$ and modifies the metabolomic profiles of the larvae of the fruit fly Drosophila melanogaster PLoS ONE 6:e25025 DOI 10.1371/journal.pone.0025025.

Koštál V, Miklas B, Doležal P, Rozsypal J, Zahradníčková H. 2014. Physiology of cold tolerance in the bark beetle, Pityogenes chalcographus and its overwintering in spruce stands. Journal of Insect Physiology 63:62-70 DOI 10.1016/j.jinsphys.2014.02.007.

Lester JD, Irwin JT. 2012. Metabolism and cold tolerance of overwintering adult mountain pine beetles (Dendroctonus ponderosae): Evidence of facultative diapause? Journal of Insect Physiology 58:808-815 DOI 10.1016/j.jinsphys.2012.03.003.

Li A, Denlinger DL. 2008. Rapid cold hardening elicits changes in brain protein profiles of the flesh fly, Sarcophaga crassipalpis. Insect Molecular Biology 17:565-572 DOI 10.1111/j.1365-2583.2008.00827.x.

Li B, Dewey CN. 2011. RSEM: accurate transcript quantification from RNA-Seq data with or without a reference genome. Bmc Bioinformatics 12:323 DOI 10.1186/1471-2105-12-323.

Li R, Wang YT, Jiang GF. 2019. The transcriptome analysis of the bamboo grasshopper provides insights into hypothermic stress acclimation. International Journal of Biological Macromolecules 134:237-246 DOI 10.1016/j.ijbiomac.2019.05.002.

Li W, Godzik A. 2006. Cd-hit: a fast program for clustering and comparing large sets of protein or nucleotide sequences. Bioinformatics (Oxford, England) 22:1658-1659 DOI 10.1093/bioinformatics/btl158.

Lindeman AA, Yack JE. 2015. What is the password? Female bark beetles (Scolytinae) grant males access to their galleries based on courtship song. Behavioural Processes 115:123-131 DOI 10.1016/j.beproc.2015.03.009.

Livak KJ, Schmittgen TD. 2001. Analysis of relative gene expression data using real-time quantitative PCR and the $2^{-\triangle} \triangle \mathrm{t}$ Method. Methods 25:402-408 DOI 10.1006/meth.2001.1262.

MacMillan HA, Knee JM, Liu Z, Xu B, Guo Y, Raffa K F, Sun J. 2017. Gallery and acoustic traits related to female body size mediate male mate choice in a bark beetle. Animal Behaviour 125:41-50 DOI 10.1016/j.anbehav.2017.01.002.

Dennis AB, Udaka H, Marshall KE, Merritt TJ, Sinclair BJ. 2016. Cold acclimation wholly reorganizes the Drosophila melanogaster transcriptome and metabolome. Scientific Reports 6:28999 DOI 10.1038/srep28999.

Malone JH, Oliver B. 2011. Microarrays, deep sequencing and the true measure of the transcriptome. Bmc Biology 9:34 DOI 10.1186/1741-7007-9-34.

Martinson EO, Peyton J, Kelkar YD, Jennings EC, Benoit JB, Werren JH, Denlinger DL. 
2019. Genome and ontogenetic-based transcriptomic analyses of the flesh fly, Sarcophaga bullata. G3 (Bethesda) 9:1313-1320 DOI 10.1534/g3.119.400148.

Michaud MR, Denlinger DL. 2007. Shifts in the carbohydrate, polyol, and amino acid pools during rapid cold-hardening and diapause-associated cold-hardening in flesh flies (Sarcophaga crassipalpis): a metabolomic comparison. Journal of Comparative Physiology B 177:753-763 DOI 10.1007/s00360-007-0172-5.

Oppenheim SJ, Baker RH, Simon S, DeSalle R. 2015. We can't all be supermodels: the value of comparative transcriptomics to the study of non-model insects. Insect Molecular Biology 24:139-154 DOI 10.1111/imb.12154.

Parker DJ, Vesala L, Laiho A, Hoikkala A, Kankare M, Ritchie MG. 2015. How consistent are the transcriptome changes associated with cold acclimation in two species of the Drosophila virilis group? Heredity: An International Journal of Genetics 115:13-21DOI 10.1038/hdy.2015.6.

Parker DJ, Wiberg R, Trivedi U, Tyukmaeva VI, Gharbi K, Butlin RK, Hoikkala A, Kankare M, Ritchie MG. 2018. Inter and intraspecific genomic divergence in Drosophila montana shows evidence for cold adaptation. Genome Biology and Evolution 10:2086-2101 DOI 10.1093/gbe/evy147.

Pei J, Li C, Ren L, Zong S. 2020. Factors influencing cold hardiness during overwintering of Streltzoviella insularis (Lepidoptera: Cossidae). Journal of Economic Entomology. DOI 10.1093/jee/toaa032.

Pfister TD, Storey KB. 2006. Insect freeze tolerance: Roles of protein phosphatases and protein kinase A. Insect Biochemistry and Molecular Biology 36:18-24 DOI 10.1016/j.ibmb.2005.10.002.

\section{Poupardin R, Schöttner K, Korbelová J, Provazník J, Doležel D, Pavlinic D, Benes V,} Koštál V. 2015. Early transcriptional events linked to induction of diapause revealed by RNAseq in larvae of drosophilid fly, Chymomyza costata. BMC Genomics 16(1) DOI 10.1186/s12864-015-1907-4.

Raffa KF, Hanshew AS, Mason CJ. 2016. Contributions by host trees and insect activity to bacterial communities in Dendroctonus valens (Coleoptera: Curculionidae) galleries, and their high overlap with other microbial assemblages of bark beetles. Environmental Entomology 45:348-356 DOI 10.1093/ee/nvv184.

Reddy AS, Ali GS, Celesnik H, Day IS. 2011. Coping with stresses: roles of calcium- and calcium/calmodulin-regulated gene expression. Plant Cell 23:2010-2032 DOI 10.1105/tpc.111.084988.

Régnière J, Bentz B. 2007. Modeling cold tolerance in the mountain pine beetle, Dendroctonus ponderosae. Journal of Insect Physiology 53:559-572 DOI 10.1016/j.jinsphys.2007.02.007. 


\section{Robert JA, Bonnett T, Pitt C, Spooner LJ, Fraser J, Yuen MMS, Keeling CI, Bohlmann J,} Huber DPW. 2016. Gene expression analysis of overwintering mountain pine beetle larvae suggests multiple systems involved in overwintering stress, cold hardiness, and preparation for spring development. PeerJ 4:e2109 DOI 10.7717/peerj.2109.

Salt, WR. 1961. Principles of Insect Cold-Hardiness. Annual Review of Entomology 6:55-74 DOI 10.1146/annurev.en.06.010161.000415.

Seppey M, Manni M, Zdobnov EM. 2019. BUSCO: Assessing Genome Assembly and Annotation Completeness. Gene Prediction 1962:227-245 DOI 10.1007/978-1-4939-9173$0 \_14$.

Simao FA, Waterhouse RM, Ioannidis P, Kriventseva EV, Zdobnov EM. 2015. BUSCO: assessing genome assembly and annotation completeness with single-copy orthologs. Bioinformatics 31:3210-3212 DOI 10.1093/bioinformatics/btv351.

Sinclair BJ. 2015. Linking energetics and overwintering in temperate insects. Journal of Thermal Biology 54:5-11DOI 10.1016/j.jtherbio.2014.07.007.

Smith-Unna R, Boursnell C, Patro R, Hibberd JM, Kelly S. 2016. TransRate: reference-free quality assessment of de novo transcriptome assemblies. Genome Research 26:1134-1144 DOI 10.1101/gr.196469.115.

Stetina T, Hula, P, Moos M, Simek P, smilauer P, Kostal V. 2018. Recovery from supercooling, freezing, and cryopreservation stress in larvae of the drosophilid fly, Chymomyza costata. Scientific Reports 8(1) DOI 10.1038/s41598-018-22757-0.

Storey JM, Storey KB, Zhang GJ. 2011. Chaperone proteins and winter survival by a freeze tolerant insect. Journal of Insect Physiology 57:1115-1122 DOI 10.1016/j.jinsphys.2011.02.016.

Storey KB, Storey JM. 1990. Metabolic rate depression and biochemical adaptation in anaerobiosis, hibernation and estivation. Quarterly Review of Biology 65:145-174 DOI $10.1086 / 416717$.

Storey KB, Storey JM. 2011. Heat shock proteins and hypometabolism: adaptive strategy for proteome preservation. Research and Reports in Biology 2:57-68 DOI 10.2147/RRB.S13351.

Sun J, Lu M, Gillette NE, Wingfield MJ. 2013. Red Turpentine Beetle: Innocuous Native Becomes Invasive Tree Killer in China. Annual Review of Entomology 58:293-311 DOI 10.1146/annurev-ento-120811-153624.

Sun J, Miao Z, Zhang Z, Zhang Z, Gillette NE. 2004. Red Turpentine Beetle, Dendroctonus valens LeConte (Coleoptera: Scolytidae), Response to Host Semiochemicals in China. Environmental Entomology 33:206-212 DOI 10.1603/0046-225X-33.2.206.

Tang B, Liu X, Shi Z, Shen Q, Xu Y, Wang S, Zhang F, Wang S. 2017. Transcriptome 
analysis and identification of induced genes in the response of Harmonia axyridis to cold hardiness. Comparative biochemistry and physiology, Part D. Genomics \& proteomics 22:78-89 DOI 10.1016/j.cbd.2017.01.004.

Teets NM, Peyton JT, Ragland GJ, Colinet H, Renault D, Hahn DA, Denlinger DL. 2012. Combined transcriptomic and metabolomic approach uncovers molecular mechanisms of cold tolerance in a temperate flesh fly. Physiological Genomics 44:764-777 DOI 10.1152/physiolgenomics.00042.2012.

Teets NM, Peyton JT, Colinet H, Renault D, Kelley JL, Kawarasaki Y, Lee RE, Denlinger DL. 2012. Gene expression changes governing extreme dehydration tolerance in an Antarctic insect. Proceedings of the National Academy of Sciences 109:20744-20749 DOI 10.1073/pnas.1218661109.

Terlecky SR, Chiang HL, Olson TS, Dice JF. 1992. Protein and peptide binding and stimulation of in vitro lysosomal proteolysis by the $73-\mathrm{kDa}$ heat shock cognate protein. Journal of Biological Chemistry 267:9202-9209.

Tomalty HE, Graham LA, Eves R, Gruneberg AK, Davies PL. 2019. Laboratory-scale isolation of insect antifreeze protein for cryobiology. Biomolecules 9:180 DOI 10.3390/biom9050180.

Toxopeus J, Des Marteaux LE, Sinclair BJ. 2019. How crickets become freeze tolerant: The transcriptomic underpinnings of acclimation in Gryllus veletis. Comparative Biochemistry and Physiology Part D: Genomics and Proteomics 29:55-66 DOI 10.1016/j.cbd.2018.10.007.

Toxopeus J, Koštál V, Sinclair BJ. 2019. Evidence for non-colligative function of small cryoprotectants in a freeze-tolerant insect. Proceedings. Biological sciences 286:20190050 DOI 10.1098/rspb.2019.0050.

Toxopeus J, Sinclair BJ. 2018. Mechanisms underlying insect freeze tolerance. Biological Reviews 93:1891-1914 DOI 10.1111/brv.12425.

Tusong K, Liu X, Guo X, Ma J, Meng S. 2017. Comparative analysis of the transcriptome of the overwintering desert beetle Microdera punctipennis. Cryobiology: International Journal of Low Temperature Biology and Medicine 78:80-89 DOI 10.1016/j.cryobiol.2017.06.009.

Untergasser A, Nijveen H, Rao X, Bisseling T, Geurts R, Leunissen JA. 2007. Primer3Plus, an enhanced web interface to Primer3. Nucleic Acids Research 35:W71-W74 DOI 10.1093/nar/gkm306.

Verheyen J, De Block M, Temmerman K, Stoks R. 2018. Voltinism-associated differences in winter survival across latitudes: integrating growth, physiology, and food intake. Oecologia 186:919-929 DOI 10.1007/s00442-018-4079-5.

Vesala L, Salminen TS, Laiho A, Hoikkala A, Kankare M. 2012. Cold tolerance and 
cold-induced modulation of gene expression in two Drosophila virilis group species with different distributions. Insect Molecular Biology 21:107-118 DOI 10.1111/j.13652583.2011.01119.x.

Wang B, Sun J, Lu M, Salcedo C. 2012. Mutual interactions between an invasive bark beetle and its associated fungi. Bulletin of Entomological Research 102:71-77 DOI 10.1017/S000748531100037X.

Wang J, Gao G, Zhang R, Dai L, Chen H. 2016. Metabolism and cold tolerance of Chinese white pine beetle Dendroctonus armandi (Coleoptera: Curculionidae: Scolytinae) during the overwintering period. Agricultural and Forest Entomology 19:10-22 DOI 10.1111/afe.12176.

Wang S, Sun J, Zhou F, Wang B, Lou Q, Xu L, Lu M. 2017. Bacterial volatile ammonia regulates the consumption sequence of D-pinitol and D-glucose in a fungus associated with an invasive bark beetle. The ISME journal emultidisciplinary journal of microbial ecology 11:2809-2820 DOI 10.1038/ismej.2017.131.

Wang XC, Zhao QY, Ma CL, Zhang ZH, Cao HL, Kong YM, Yue C, Hao XY, Chen L, Ma JQ, Jin JQ, Li X, Yang YJ. 2013. Global transcriptome profiles of Camellia sinensis during cold acclimation. Bmc Genomics 14:415 DOI 10.1186/1471-2164-14-415.

Wang XR, Wang C, Ban FX, Zhu DT, Liu SS, Wang XW. 2019. Genome-wide identification and characterization of HSP gene superfamily in whitefly (Bemisia tabaci) and expression profiling analysis under temperature stress. Insect Science 26:44-57 DOI 10.1111/17447917.1020.03.040.

Waterhouse RM, Seppey M, Simao FA, Manni M, Ioannidis P, Klioutchnikov G, Kriventseva, EV, Zdobnov EM. 2018. BUSCO Applications from Quality Assessments to Gene Prediction and Phylogenomics. Molecular Biology and Evolution 35:543-548 DOI 10.1093/molbev/msx319.

Wharton DA. 2011. Cold tolerance of New Zealand alpine insects. Journal of Insect Physiology 57:1090-1095 DOI 10.1016/j.jinsphys.2011.03.004.

Xiang H, Yang X, Ke L, Hu Y. 2020. The properties, biotechnologies, and applications of antifreeze proteins. International Journal of Biological Macromolecules 153:661-675 DOI 10.1016/j.ijbiomac.2 of America 107:152-157 DOI 10.1603/AN13066.

Xie C, Mao X, Huang J, Ding Y, Wu J, Dong S, Kong L, Gao G, Li C, Wei L. 2011. KOBAS 2.0: a web server for annotation and identification of enriched pathways and diseases. Nucleic Acids Research 39:W316-W322 DOI 10.1093/nar/gkr483.

Xu BB, Liu Z, Sun JH. 2014. Instar numbers, development, flight period, and fecundity of Dendroctonus valens (Coleoptera: Curculionidae: Scolytinae) in China. Annals of the Entomological Society2505. 
875 Xu BB, Liu ZD, Sun JH. 2014. The effects of $\alpha$-pinene on the feeding performance and pheromone production of Dendroctonus valens. Entomologia Experimentalis Et Applicata 150:269-278 DOI 10.1111/eea.12161.

Xu K, Niu Q, Zhao H, Du Y, Jiang Y. 2017. Transcriptomic analysis to uncover genes affecting cold resistance in the Chinese honey bee (Apis cerana cerana). PLOS ONE 12:e0179922 DOI 10.1371/journal.pone.0179922.

Yan Z, Sun J, Don O, Zhang Z. 2005. The red turpentine beetle, Dendroctonus valens LeConte (Scolytidae): an exotic invasive pest of pine in China. Biodiversity and Conservation 14:1735-1760 DOI 10.1007/s10531-004-0697-9.

Yang ZQ, Wang XY, Zhang YN. 2014. Recent advances in biological control of important native and invasive forest pests in China. Biological Control: Theory and Application in Pest Management 68:117-128 DOI 10.1016/j.biocontrol.2013.06.010.

Yu SH, Yang P, Sun T, Qi Q, Wang XQ, Chen XM, Feng Y, Liu BW. 2016. Transcriptomic and proteomic analyses on the supercooling ability and mining of antifreeze proteins of the Chinese white wax scale insect. Insect Science 23:430-437 DOI 10.1111/1744-7917.12320.

Zhang L, Liu Y, Yao J, Wang B, Huang B, Li Z, Fan M, Sun J. 2011. Evaluation of Beauveria bassiana (Hyphomycetes) isolates as potential agents for control of Dendroctonus valens. Insect Science 18:209-216 DOI 10.1111/j.1744-7917.2010.01361.x.

Zhang Y, Wu H, Xie J, Jiang R, Deng C, Pang H. 2015. Transcriptome responses to heat- and cold-stress in ladybirds (Cryptolaemus montrouzieri Mulasnt) analyzed by deep-sequencing. Biological Research 48(1) DOI 10.1186/s40659-015-0054-3.

Zhang Z, Wang H, Kong X, Chen H. 2010. Antennal morphology and sensilla ultrastructure of Dendroctonus valens LeConte (Coleoptera: Curculionidae, Seolytinae), an invasive forest pest in China. Micron: The international research and review journal for microscopy 41:735-741 DOI 10.1016/j.micron.2010.06.007.

Zhao J, Yang Z, Gregoire, JC. 2009. The cold-hardiness of Dendroctonus valens (Coleoptera, Scolytidae) and Rhizophagusgrandis (Coleoptera, Rhizophagidae). Journal of Environmental Entomology 31:20-28 DOI 1674-0858 (2009) 01-0020-09.

Zheng C, Zhao D, Xu Y, Shi F, Zong S, Tao J. 2020. Reference gene selection for expression analyses by qRT-PCR in Dendroctonus valens. Insects 11:328 DOI 10.3390/insects11060328.

Zhou X, Shan Y, Tan Y, Zhang Z, Pang B. 2019. Comparative Analysis of Transcriptome Responses to Cold Stress in Galeruca daurica (Coleoptera: Chrysomelidae). Journal of insect science (Online) 19:8 DOI 10.1093/jisesa/iez109. 


\section{Table $\mathbf{1}$ (on next page)}

Statistical summary of sequencing data for 12 cDNA samples from $D$. valens.

In sample names, $\mathrm{CL}$ indicates larvae collected in January, NL indicates larvae collected in May, CA indicates adults collected in January, and NA indicates adults collected in May. 
Table 1:

2 Statistical summary of sequencing data for 12 cDNA samples from D. valens.

\begin{tabular}{|c|c|c|c|c|c|c|}
\hline Sample & $\begin{array}{l}\text { Raw } \\
\text { Bases }\end{array}$ & $\begin{array}{l}\text { Clean } \\
\text { bases }\end{array}$ & $\begin{array}{l}\text { Error rate } \\
(\%)\end{array}$ & $\begin{array}{l}\text { Q20 } \\
(\%)\end{array}$ & $\begin{array}{l}\text { Q30 } \\
(\%)\end{array}$ & $\begin{array}{l}\text { GC content } \\
(\%)\end{array}$ \\
\hline $\mathrm{CL}_{-} 1$ & 7625015592 & 7435581614 & 0.0258 & 97.81 & 93.21 & 42.31 \\
\hline CL_2 & 6606424254 & 6408262061 & 0.0258 & 97.82 & 93.23 & 42.14 \\
\hline $\mathrm{CL} \_3$ & 7213969734 & 7003588404 & 0.0253 & 98.01 & 93.71 & 43.54 \\
\hline $\mathrm{NL} \_1$ & 6855725858 & 6669592971 & 0.0247 & 98.16 & 94.46 & 49.37 \\
\hline $\mathrm{NL} \_2$ & 6275942634 & 6141265236 & 0.0245 & 98.22 & 94.58 & 46.91 \\
\hline $\mathrm{NL} \_3$ & 6658060516 & 6433890287 & 0.0251 & 97.96 & 94.05 & 50.28 \\
\hline $\mathrm{CA}_{-} 1$ & 7973857302 & 7780530188 & 0.0235 & 98.61 & 95.64 & 42.34 \\
\hline CA_2 & 7687548920 & 7512555521 & 0.0237 & 98.55 & 95.48 & 42.12 \\
\hline CA_3 & 7478884134 & 7284498797 & 0.0234 & 98.67 & 95.72 & 39.96 \\
\hline NA_1 & 8155209510 & 7979742248 & 0.0235 & 98.63 & 95.62 & 38.6 \\
\hline NA_2 & 8403092318 & 8234779825 & 0.0233 & 98.71 & 95.86 & 40.07 \\
\hline NA_3 & 7578902910 & 7436520894 & 0.0236 & 98.62 & 95.59 & 38.13 \\
\hline
\end{tabular}

3 In sample names, CL indicates larvae collected in January, NL indicates larvae collected in May, CA indicates 4 adults collected in January, and NA indicates adults collected in May.

5 


\section{Table 2 (on next page)}

KEGG pathway analysis of common DEGs between sampling dates in $D$. valens larvae and adults. 
1 Table 2:

2 KEGG pathway analysis of common DEGs between sampling dates in D. valens larvae and adults

\begin{tabular}{lllc}
\hline Pathway ID & Second Category & Description & Count \\
\hline map04714 & Environmental adaptation & Thermogenesis & 20 \\
map04142 & Transport and catabolism & Lysosome & 19 \\
map00230 & Nucleotide metabolism & Purine metabolism & 18 \\
map04140 & Transport and catabolism & Autophagy - animal & 18 \\
map04024 & Signal transduction & cAMP signaling pathway & 15 \\
map00190 & Energy metabolism & Oxidative phosphorylation & 14 \\
map04530 & Cellular community - eukaryotes & Tight junction & 13 \\
map00040 & Carbohydrate metabolism & Pentose and glucuronate interconversions & 12 \\
map04141 & Folding, sorting and degradation & Protein processing in endoplasmic reticulum & 12 \\
map04210 & Cell growth and death & Apoptosis & 12 \\
map04910 & Endocrine system & Insulin signaling pathway & 12 \\
map00520 & Carbohydrate metabolism & Amino sugar and nucleotide sugar metabolism & 11 \\
map04022 & Signal transduction & cGMP-PKG signaling pathway & 11 \\
map04260 & Circulatory system & Cardiac muscle contraction & 11 \\
map00240 & Nucleotide metabolism & Pyrimidine metabolism & 10 \\
map04010 & Signal transduction & MAPK signaling pathway & 10 \\
map04145 & Transport and catabolism & Phagosome & 10 \\
map04921 & Endocrine system & Oxytocin signaling pathway & 10 \\
\hline map04723 & Nervous system & Retrograde endocannabinoid signaling & 10 \\
\hline
\end{tabular}

3 


\section{Table 3 (on next page)}

GO enrichment of common DEGs between sampling dates in $D$. valens larvae and adults. 
1 Table 3:

2 GO enrichment of common DEGs between sampling dates in D. valens larvae and adults

\begin{tabular}{|c|c|c|c|c|}
\hline GO category & $\begin{array}{l}\text { Term } \\
\text { type }\end{array}$ & GO code & Number & P-value \\
\hline carbohydrate metabolic process & $\mathrm{BP}$ & GO:0005975 & 65 & $2.63 \mathrm{E}-06$ \\
\hline external encapsulating structure organization & $\mathrm{BP}$ & GO:0045229 & 7 & $3.70 \mathrm{E}-04$ \\
\hline cell wall organization or biogenesis & $\mathrm{BP}$ & GO:0071554 & 7 & $3.70 \mathrm{E}-04$ \\
\hline cell wall organization & $\mathrm{BP}$ & GO:0071555 & 7 & $3.70 \mathrm{E}-04$ \\
\hline membrane lipid metabolic process & $\mathrm{BP}$ & GO:0006643 & 13 & $3.76 \mathrm{E}-04$ \\
\hline cell wall modification & $\mathrm{BP}$ & GO:0042545 & 4 & $1.05 \mathrm{E}-03$ \\
\hline $\begin{array}{l}\text { RNA polymerase II transcriptional preinitiation complex } \\
\text { assembly }\end{array}$ & $\mathrm{BP}$ & GO:0051123 & 4 & $1.05 \mathrm{E}-03$ \\
\hline polysaccharide catabolic process & $\mathrm{BP}$ & GO:0000272 & 6 & $1.47 \mathrm{E}-03$ \\
\hline organic acid biosynthetic process & $\mathrm{BP}$ & GO:0016053 & 18 & $1.50 \mathrm{E}-03$ \\
\hline carboxylic acid biosynthetic process & $\mathrm{BP}$ & GO:0046394 & 18 & $1.50 \mathrm{E}-03$ \\
\hline polysaccharide metabolic process & $\mathrm{BP}$ & GO:0005976 & 8 & $1.62 \mathrm{E}-03$ \\
\hline small molecule metabolic process & $\mathrm{BP}$ & GO:0044281 & 76 & $2.13 \mathrm{E}-03$ \\
\hline hydrolase activity, acting on glycosyl bonds & MF & GO:0016798 & 43 & $5.80 \mathrm{E}-07$ \\
\hline hydrolase activity, hydrolyzing O-glycosyl compounds & $\mathrm{MF}$ & GO:0004553 & 40 & $1.06 \mathrm{E}-06$ \\
\hline phosphatase activity & MF & GO:0016791 & 24 & $1.01 \mathrm{E}-04$ \\
\hline hydrolase activity & MF & GO:0016787 & 213 & $1.09 \mathrm{E}-04$ \\
\hline cellulase activity & $\mathrm{MF}$ & GO:0008810 & 6 & $1.65 \mathrm{E}-04$ \\
\hline carbohydrate binding & $\mathrm{MF}$ & GO:0030246 & 16 & $2.26 \mathrm{E}-04$ \\
\hline
\end{tabular}




$\begin{array}{lcccr}\text { catalytic activity } & \text { MF } & \text { GO:0003824 } & 396 & 2.87 \mathrm{E}-04 \\ \begin{array}{l}\text { magnesium ion binding } \\ \text { pectinesterase activity }\end{array} & \text { MF } & \text { GO:0000287 } & 13 & 8.87 \mathrm{E}-04 \\ \text { aspartyl esterase activity } & \text { MF } & \text { GO:0030599 } & 4 & 1.05 \mathrm{E}-03 \\ \text { phosphatidylinositol binding } & \text { MF } & \text { GO:0045330 } & 4 & 1.05 \mathrm{E}-03 \\ \text { phosphoric ester hydrolase activity } & \text { MF } & \text { GO:0035091 } & 12 & 1.30 \mathrm{E}-03 \\ \text { serine-type exopeptidase activity } & \text { MF } & \text { GO:0042578 } & 26 & 1.47 \mathrm{E}-03 \\ & & & \\ \end{array}$

3 


\section{Table 4(on next page)}

Common differentially expressed gene set may be related to cold tolerance.

The samples were collected in January 2019 and May 2019, and May represents the control group. Up and down mean that the gene expression is up-regulated or down-regulated in January compared with May. 
1 Table 4:

2 Common differentially expressed gene set may be related to cold tolerance

\begin{tabular}{|c|c|c|c|}
\hline Gene description & $\begin{array}{l}\text { DEG } \\
\text { number }\end{array}$ & $\begin{array}{l}\text { Larval expression } \\
\text { (number) }\end{array}$ & $\begin{array}{l}\text { Adult expression } \\
\text { (number) }\end{array}$ \\
\hline \multicolumn{4}{|c|}{ Protein folding/chaperone } \\
\hline HSP70 & 1 & up (1) & $\mathrm{up}(1)$ \\
\hline HSP68 & 1 & $\operatorname{down}(1)$ & $\operatorname{up}(1)$ \\
\hline \multicolumn{4}{|c|}{ Cytoskeleton } \\
\hline tubulin & 3 & down (3) & down (2); up (1) \\
\hline actin & 6 & down (3); up (3) & down (6) \\
\hline myosin & 3 & down (2); up (1) & down (3) \\
\hline \multicolumn{4}{|c|}{ Repair/detoxicant/antioxidant } \\
\hline DNA mismatch repair protein Mlh1 & 1 & up (1) & up (1) \\
\hline thioredoxin & 1 & up (1) & down (1) \\
\hline cytochrome P450 & 7 & down (3); up (4) & down (2); up (5) \\
\hline \multicolumn{4}{|c|}{ Signal transduction } \\
\hline guanine nucleotide-binding protein & 3 & down (2); up (1) & down (2); up (1) \\
\hline rho guanine nucleotide exchange factor & 2 & down (1); up (1) & down (2) \\
\hline calcium channel protein & 3 & up (3) & down (3) \\
\hline G kinase-anchoring protein & 1 & up (1) & up (1) \\
\hline G-protein coupled receptor Mth2 & 1 & up (1) & up (1) \\
\hline \multicolumn{4}{|c|}{ Cellular processes } \\
\hline RNA polymerases I, II, and III subunit RPABC2 & 1 & up (1) & up (1) \\
\hline \multicolumn{4}{|c|}{ Other genes } \\
\hline juvenile hormone epoxide hydrolase 1 & 1 & down (1) & down (1) \\
\hline ATP synthase subunit alpha & 1 & down (1) & down (1) \\
\hline endonuclease-reverse transcriptase & 1 & down (1) & down (1) \\
\hline E3 SUMO-protein ligase & 1 & up (1) & up (1) \\
\hline E3 ubiquitin-protein ligase & 5 & down (3); up (2) & down (4); up (1) \\
\hline cathepsin & 6 & down (6) & down (6) \\
\hline zinc finger protein & 12 & down (2); up (10) & down (1); up (11) \\
\hline NADH dehydrogenase & 2 & down (1); up (1) & down (2) \\
\hline elongation factor & 1 & down (1) & down (1) \\
\hline ribosomal protein & 2 & down (2); up (1) & down (1); up (1) \\
\hline staphylococcal nuclease domain-containing protein 1 & 1 & down (1) & down (1) \\
\hline
\end{tabular}

3 The samples were collected in January 2019 and May 2019, and May represents the control group. Up and down

4 mean that the gene expression is up-regulated or down-regulated in January compared with May.

5

6 
Figure 1

Statistical summary of the functional annotations of unigenes in public databases.

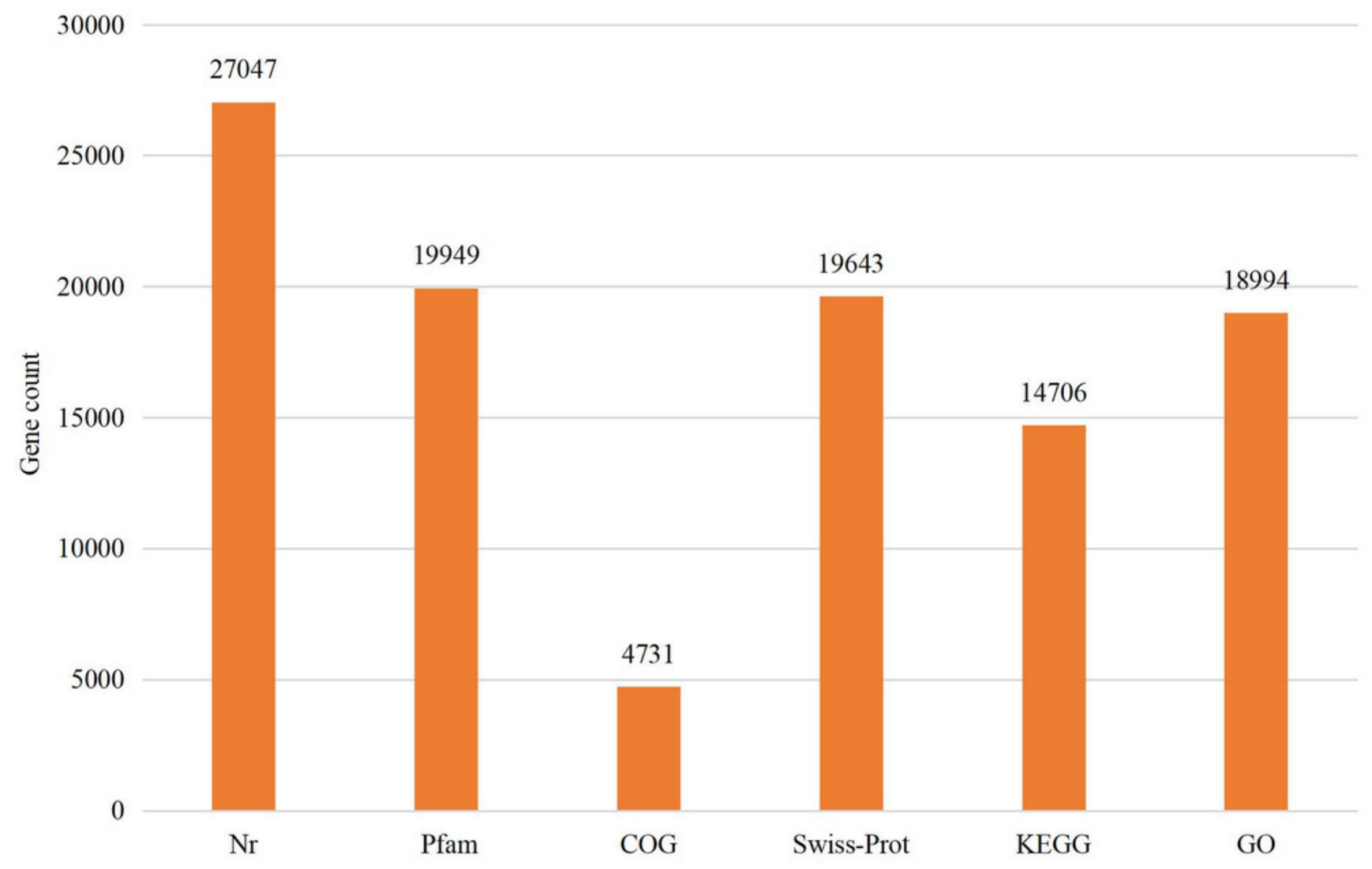


Figure 2

Pie charts showing distributions of BLAST matches for the $D$. valens unigenes with respect to $(A)$ e-values $(B)$ similarity, and $(C)$ species.

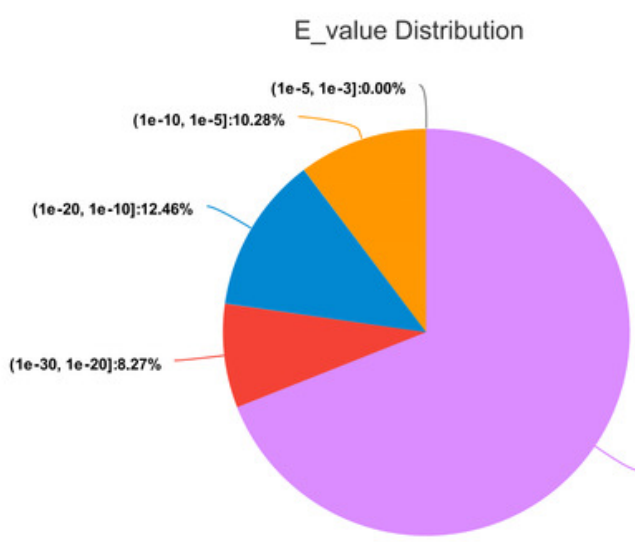

$[0,1 e-30]: 68.99 \%$

A

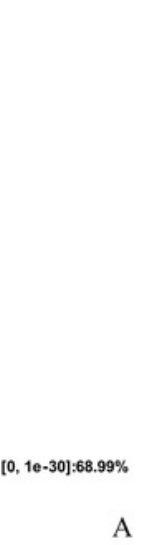

Similarity Distribution

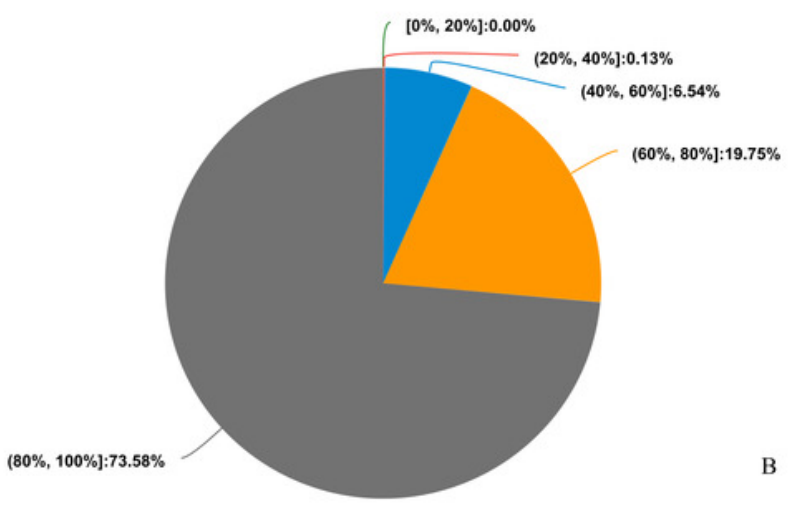

Species Distribution

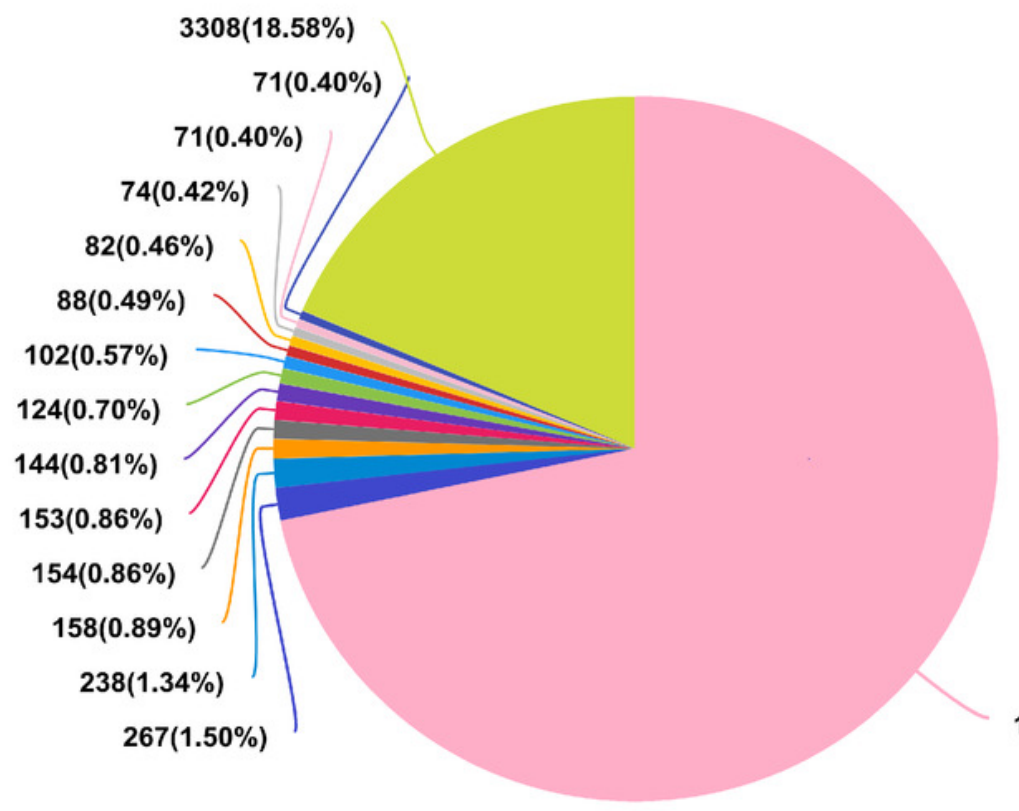

$12772(71.73 \%)$

Dendroctonus ponderosae

Anoplophora glabripennis

Eumeta japonica

Diabrotica virgifera

Tribolium castaneum

Homo sapiens

Mus musculus

Lasius niger

Leptinotarsa decemlineata

Onthophagus taurus

Larimichthys crocea

Rhagoletis zephyria

Bemisia tabaci

Nilaparvata lugens

other 


\section{Figure 3}

Distribution of DEGs between January and May in different sample types.

Distribution of DEGs between January and May in different sample types. (A) Distribution of DEGs in larvae. (B) Distribution of DEGs in adults. Volcano plot displays the relationship between the fold change and P-adjusted. Each point in the figure represents a specific unigene. Red dots represent significantly up-regulated unigenes, blue dots represent significantly down-regulated unigenes, and black dots represent unigenes that are not differentially expressed.
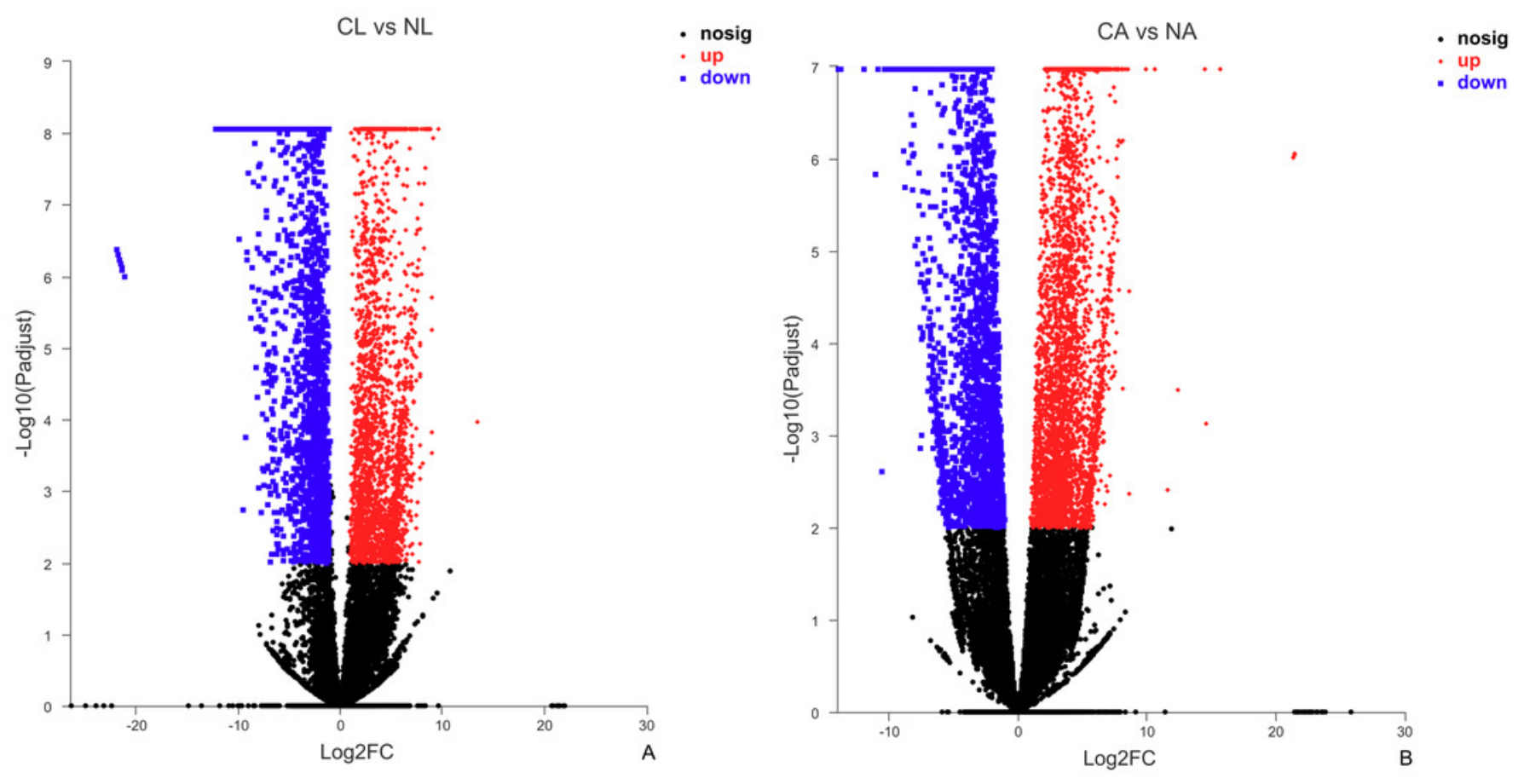
Figure 4

Venn diagram showing the number of commonly up- and down-regulated genes between the two different stages in $D$. valens.

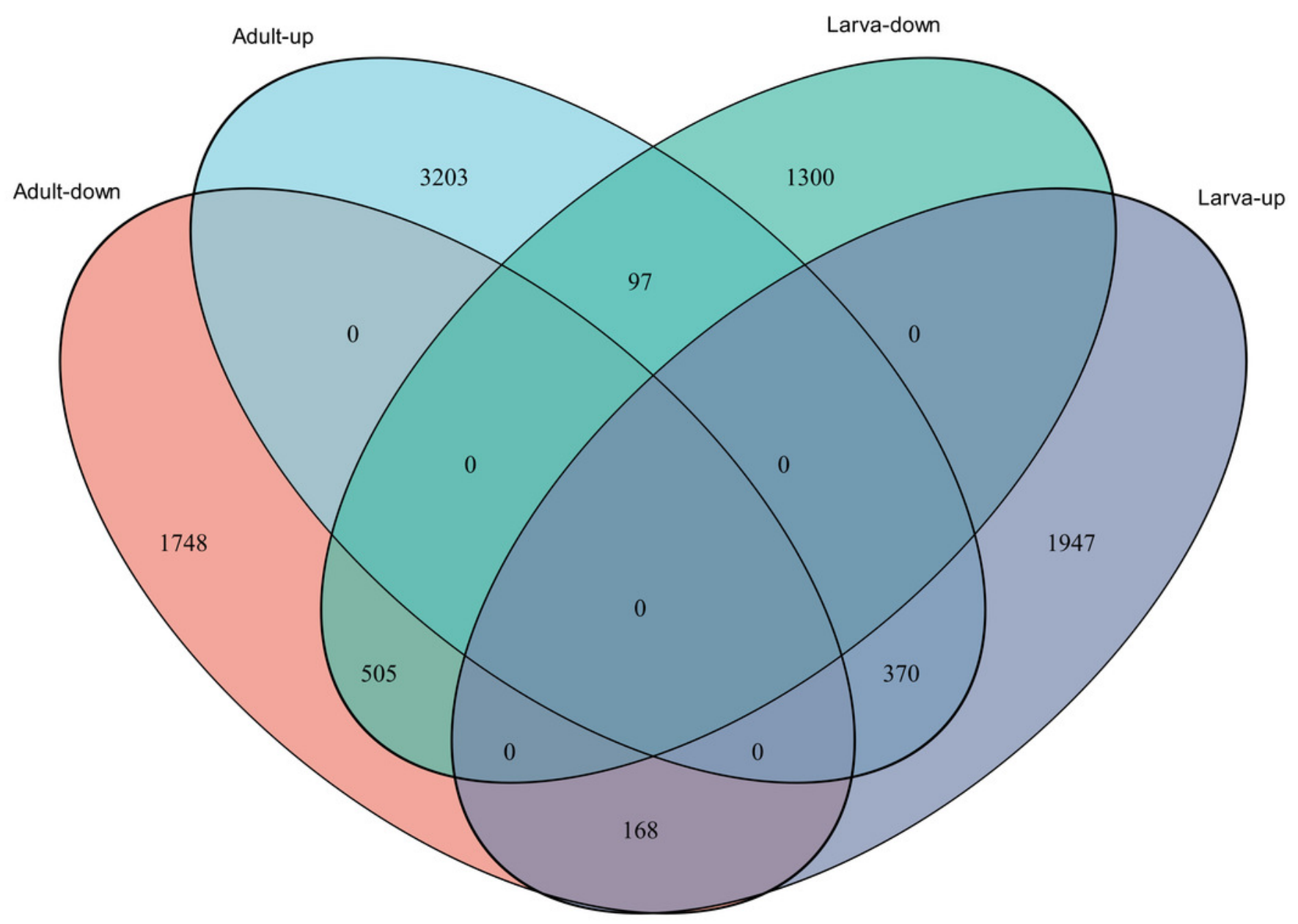




\section{Figure 5}

GO term assignments for common DEGs between collection times in $D$. valens larvae and adults.

The ordinate indicates the number of unigenes in the secondary classification. The abscissa indicates the secondary GO classification and the three colors represent the three major branches of GO (i.e., BP, CC, and MF).

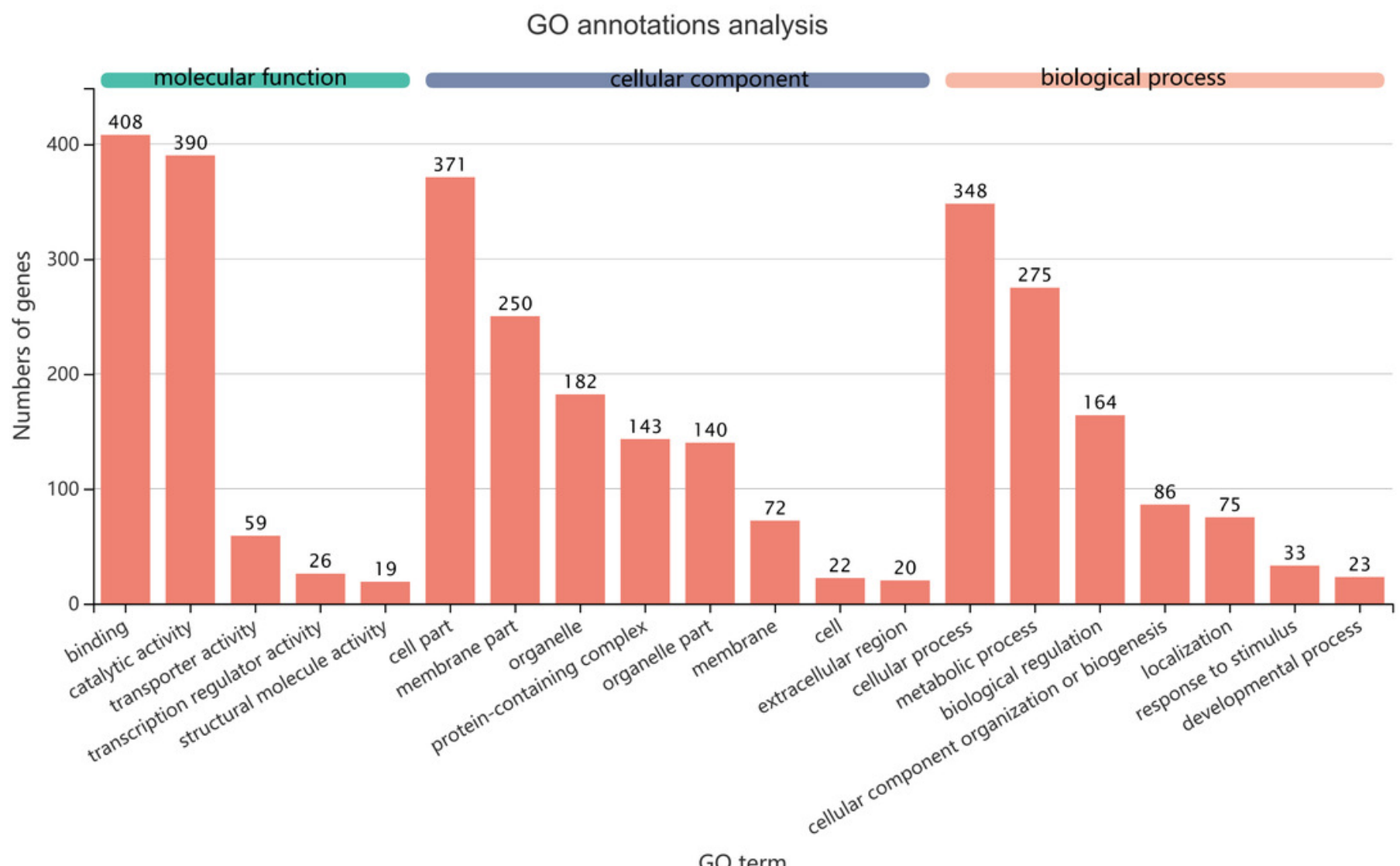


Figure 6

Heatmap of normalized FPKM values for DEGs related to cryoprotectant activity.

Heatmap of normalized FPKM values for DEGs related to cryoprotectant activity. The Z-score represents the number of standard deviations from the mean. Firebrick indicates upregulated expression, whereas navy indicates down-regulated expression. FPKM: fragments per kilobase of transcript per million fragments mapped; CL: Larva in January; NL: Larva in May; CA: Adult in January; NA: Adult in May.
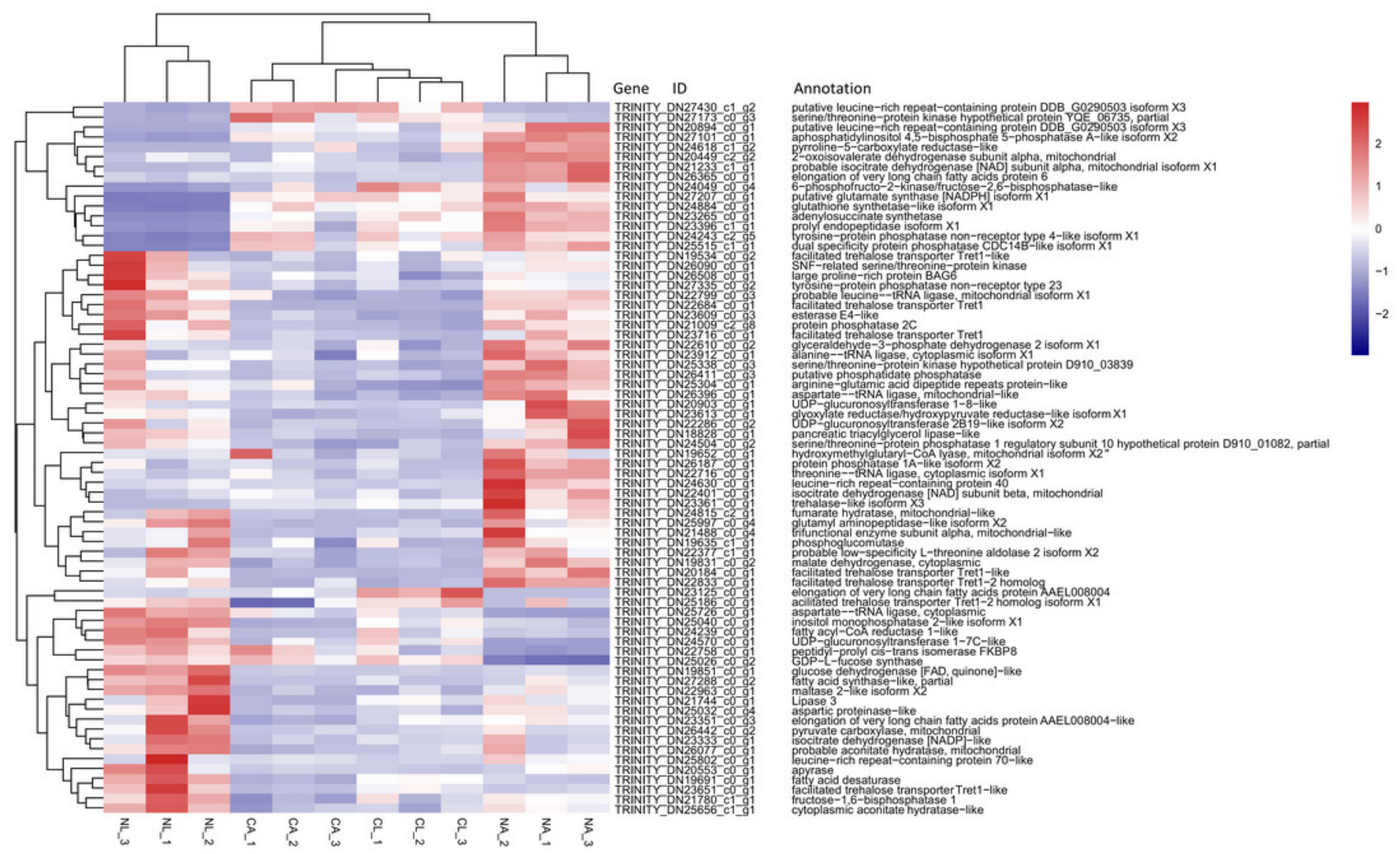


\section{Figure 7}

Validation expression patterns in $D$. valens adults by qPCR.

The samples were collected in January 2019 and May 2019. (A)staphylococcal nuclease domain-containing protein 1; (B) ATP synthase subunit alpha; (C) protein phosphatase 1A; (D) fructose-1,6-bisphosphatase 1; (E) glyceraldehyde-3-phosphate dehydrogenase 2; (F) putative glutamate synthase; (G) unknown; (H) cathepsin L1; (I) probable low-specificity Lthreonine aldolase 2; (J) E3 ubiquitin-protein ligase; (K) fatty acid desaturase; (L) serine/threonine-protein kinase; (M) uncharacterized protein; (N) uncharacterized protein. The abscissa indicates different collection dates. The left ordinate represents the qPCR-based expression levels and the right ordinate represents the RNA-seq-based expression levels. The error bar refers to SEM and the $\mathrm{N}$ value represents three repetitions. FPKM: fragments per kilobase of transcript per million fragments mapped. 

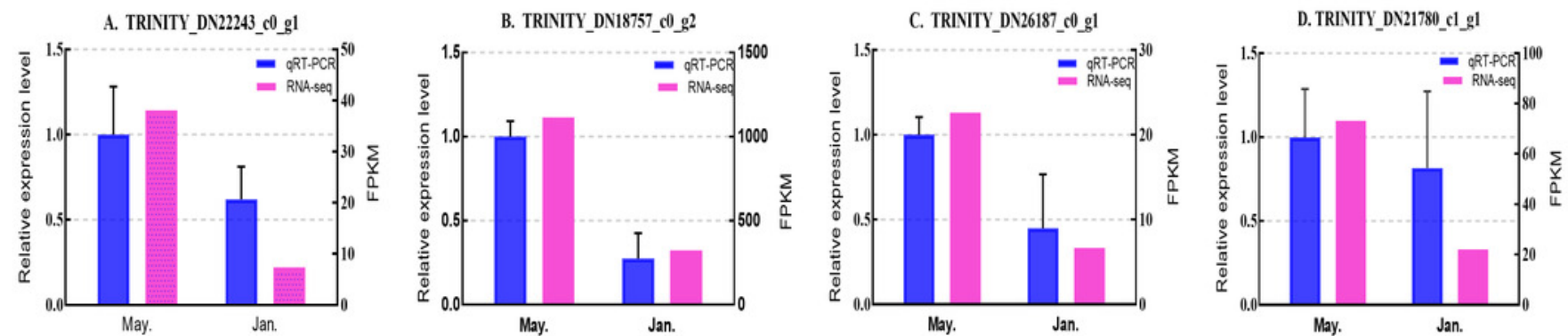

E. TRINITY_DN22610_C0_g2

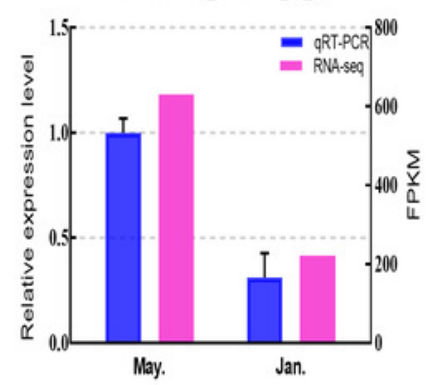

F. TRINITY DN27207 e0 gl

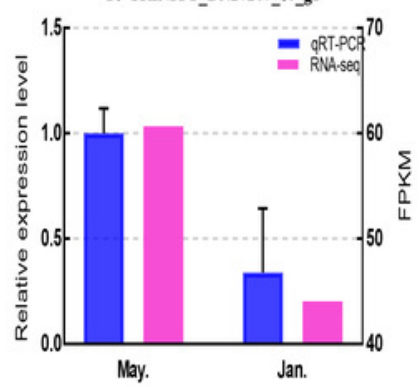

J. TRINITY_DN25425_c0_g4

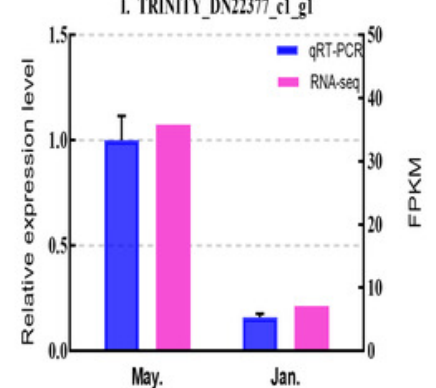

I. TRINITY DN20229_c0 gl

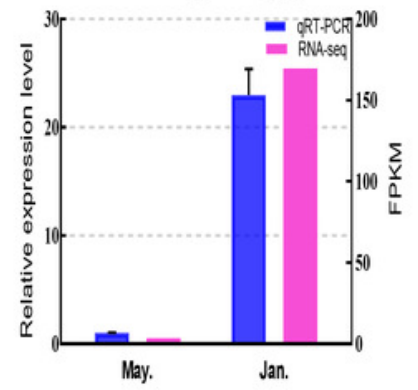

N. TRINITY_DN22439_c0 g5

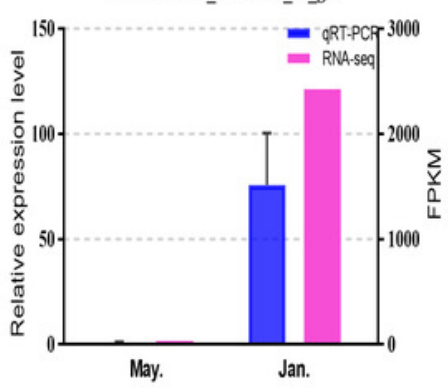

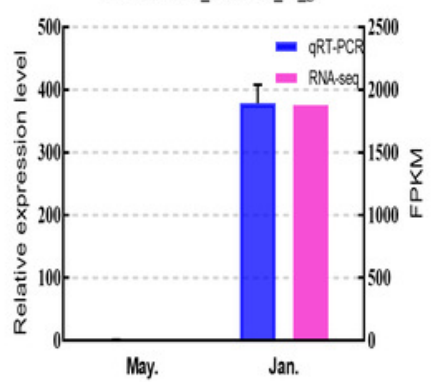

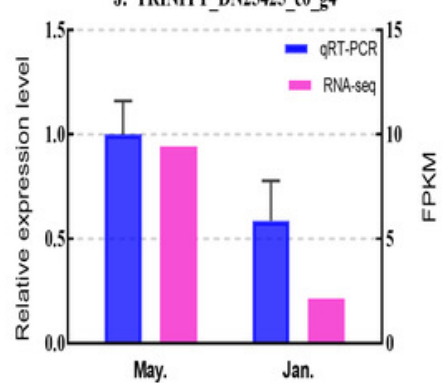

G. TRINITY DN27537 c0 g1

K. TRINITY_DN19691_C0 gl

H. TRINITY DN18927 c0 gl

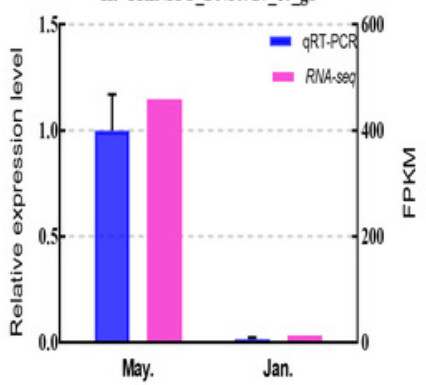

L. TRINITY_DN26090_c0_gl
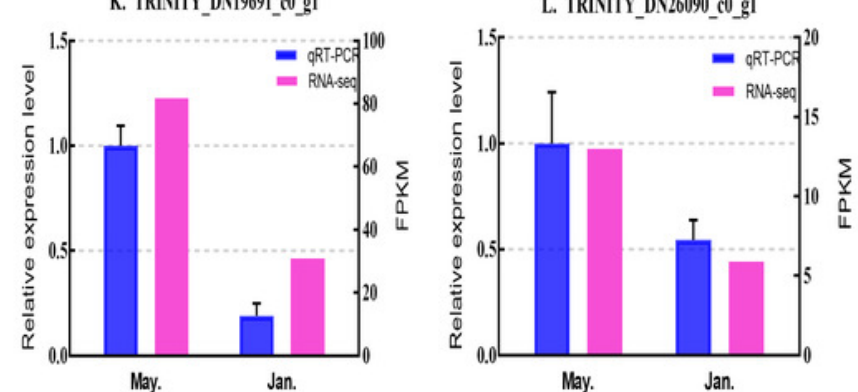


\section{Figure 8}

Validation expression patterns in $D$. valens larvae by qPCR.

The samples were collected in January 2019 and May 2019. (A)staphylococcal nuclease domain-containing protein 1; (B) ATP synthase subunit alpha; (C) protein phosphatase 1A; (D) fructose-1,6-bisphosphatase 1; (E) glyceraldehyde-3-phosphate dehydrogenase 2; (F) putative glutamate synthase; (G) unknown; (H) cathepsin L1; (I) probable low-specificity Lthreonine aldolase 2; (J) E3 ubiquitin-protein ligase; (K) fatty acid desaturase; (L) serine/threonine-protein kinase; (M) uncharacterized protein; (N) uncharacterized protein. The abscissa indicates different collection dates. The left ordinate represents the qPCR-based expression levels and the right ordinate represents the RNA-seq-based expression levels. The error bar refers to SEM and the $\mathrm{N}$ value represents three repetitions. FPKM: fragments per kilobase of transcript per million fragments mapped. 
A. TRINITY_DN22243_c0 g1

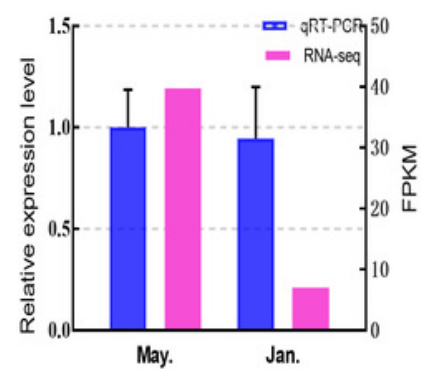

E. TRINITY_DN22610_C0_g2

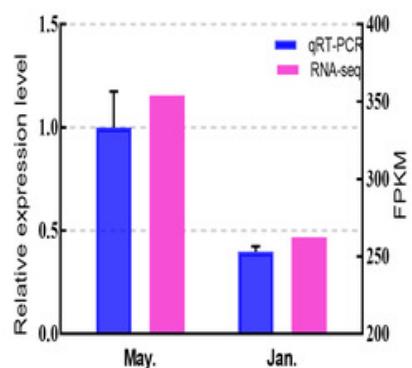

I. TRINITY_DN22377_1_g1

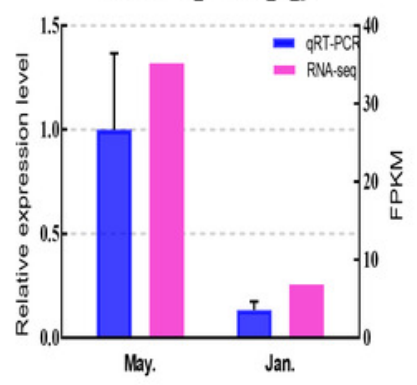

I. TRINTTY_DN20229_0 g1

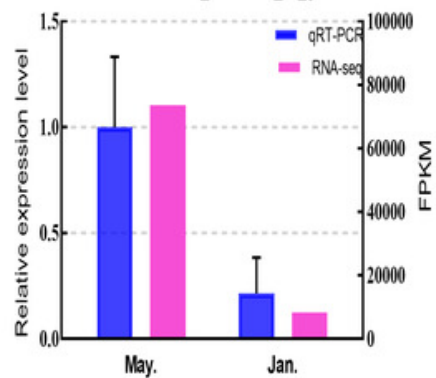

B. TRINITY_DN18757_01_g2

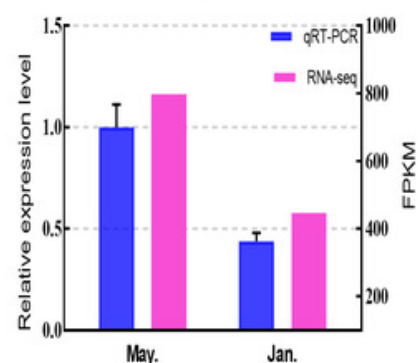

F. TRINITY_DN27207_c0_gl

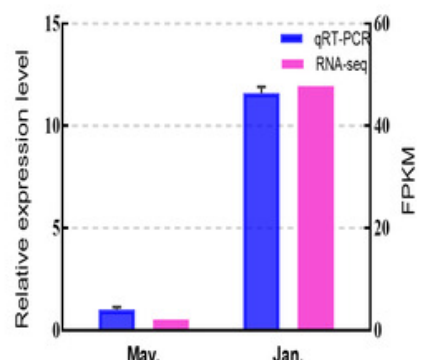

J. TRINITY_DN25425_c0 gst

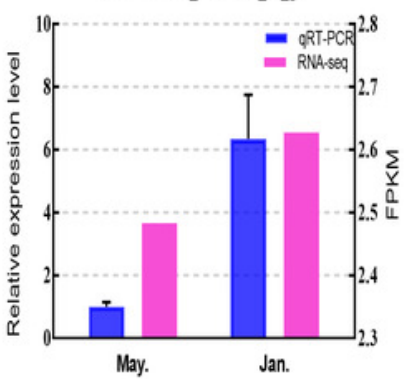

N. TRINITY_DN22439_c0 g5

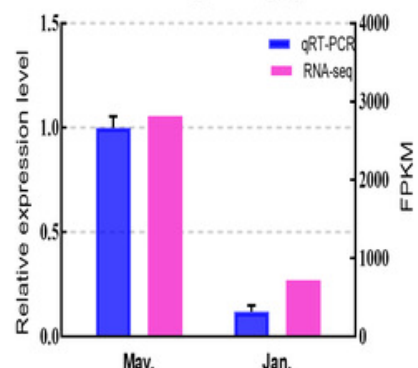

C. TRINITY_DN26187_c0 gl

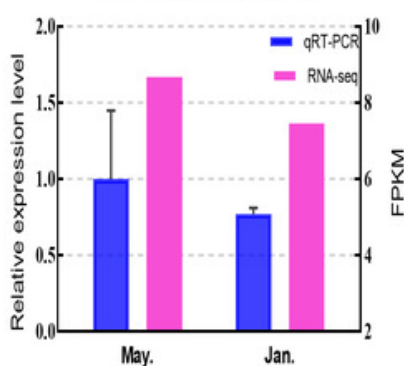

G. TRINITY DN27537_c0 g1

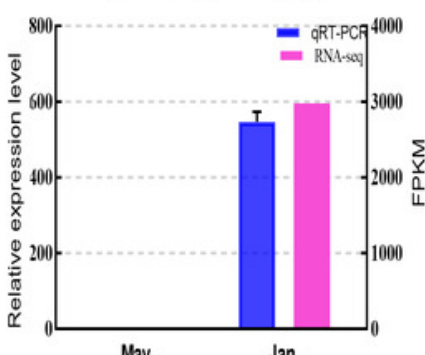

K. TRINITY_DN19691_00_g1
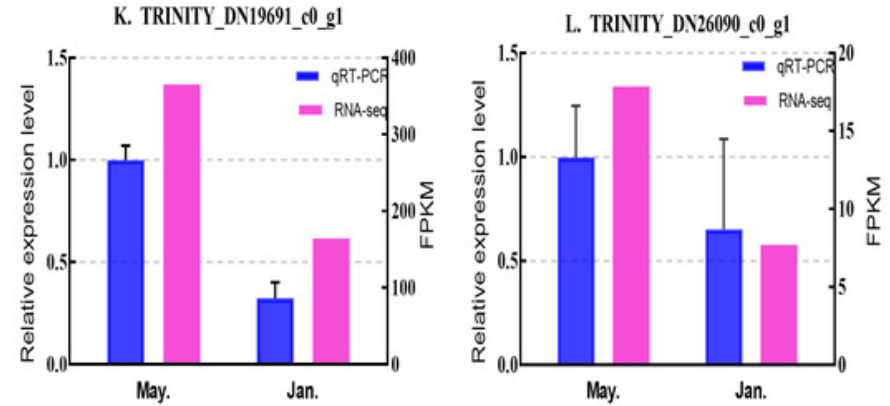

D. TRINITY_DN21780_cl_g1

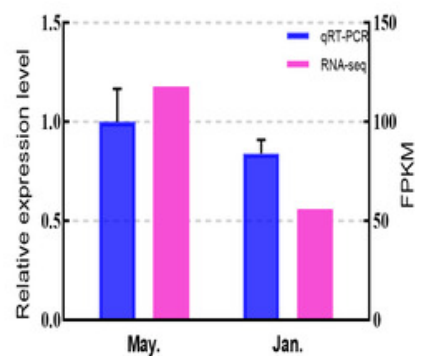

H. TRINITY_DN18927_c0_g1

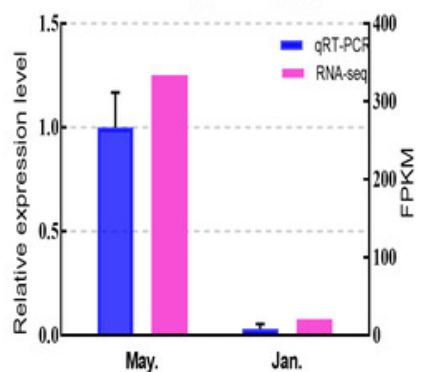

May. Jan 